\title{
8. NEONATAL INTENSIVE CARE
}

The information presented in this chapter was obtained from the Neonatal Intensive Care Units' (NICUS) Data Collection (see Chapter 3, Data sources).

\section{Registration rate}

There were 2,257 infants registered in NICUS in 2005. The most common reasons for registration of an infant were assisted ventilation for four hours or more (47.4 percent) and gestational age less than 29 weeks (16.2 percent). Infants generally met more than one of the registration criteria.

The NICUS registration rate in 2005 was 23.8 per 1,000 livebirths, which decreased slightly since 2004 (24.8 per 1,000 live births). Table 65 shows the registration rate according to the mothers' health area of residence. The relatively low registration rates from the health areas adjoining the New South Wales border reflect the fact that some infants are preferentially referred interstate. The registration rate in health areas with low numbers of births should be interpreted with caution.

Eighty-three of the 2,257 infants (3.7 per cent) registered in NICUS were born to Aboriginal or Torres Strait Islander mothers. There were 2,558 livebirths to Aboriginal or Torres Strait Islander women recorded by the NSW and ACT Midwives Data Collections for 2005. The registration rate for these infants was 32.4 per 1,000 livebirths and has decreased since 2004. Seventy-eight of the 2,070 mothers (3.8 per cent) were Aboriginal or Torres Strait Islander, of whom 17 (21.4 per cent) were residents of the Greater Western and North Coast Health Areas (Table 66). Seventeen of the 349 mothers ( 4.9 per cent) of infants less than 29 weeks and/or less than 1,000 grams were Aboriginal or Torres Strait Islander.

\section{Maternal characteristics}

There were 2,070 mothers of the 2,257 infants registered in NICUS during 2005. Nearly 90 per cent of the mothers were residents of the Sydney South West, Sydney West, Hunter \& New England, Northern Sydney \& Central Coast and South Eastern Sydney \& Illawarra Health Areas. The distribution of the mothers' health area of residence for infants less than 29 weeks and/or less than 1,000 grams was similar to those for the whole group. Of the 348 mothers of infants in this group just over three quarters (78.7 per cent) were residents of the Sydney South West, Sydney West, Hunter \& New England, Northern Sydney \& Central Coast, and South Eastern Sydney \& Illawarra Health Areas.

The age of mothers of NICUS infants ranged from 15 to 54 years, with a mean age of 29.9 years. The mean maternal age was similar across all gestational age groups and has remained constant since 1992. In 2005, 23.2 percent of mothers were aged 35 years or more (range 13.7 per cent in 1992 to 23.2 per cent in 2005). In 2005, 4.9 per cent of mothers were aged less than 20 years (range 4.7 per cent in 2004 to 6.8 per cent in 2000) (Table 67). The health area of residence with the highest proportion of teenage mothers was Hunter \& New England.

There were 1,804 mothers (87.1 per cent) who had an antenatal complication. The most common antenatal complications were preterm labour (43.6 per cent), pregnancy induced hypertension (16.9 per cent), fetal distress (17.2 per cent), antepartum haemorrhage (17.2 per cent), and intrauterine growth restriction (9.6 percent). Antenatal complications were more frequent in mothers delivering at less than 37 weeks compared with at term. Even so, 56 per cent of mothers giving birth at term had an antenatal complication (Table 68).

\section{TABLE 65}

NICUS REGISTRATIONS BY HEALTH AREA OF RESIDENCE, NSW \& ACT 2005

\begin{tabular}{|c|c|c|c|c|}
\hline Health Area & No. & $\%$ & $\begin{array}{c}\text { Total NSW \& ACT } \\
\text { live births } \\
\text { No. }\end{array}$ & $\begin{array}{l}\text { Registrants } \\
\text { per } 1,000 \text { live births }\end{array}$ \\
\hline Sydney South West & 474 & 21.0 & 19846 & 23.9 \\
\hline South Eastern Sydney \& Illawarra & 295 & 13.1 & 14770 & 20.0 \\
\hline Sydney West & 435 & 19.3 & 17246 & 25.2 \\
\hline Northern Sydney \& Central Coast & 263 & 11.7 & 13876 & 19.0 \\
\hline Hunter \& New England & 361 & 16.0 & 10497 & 34.4 \\
\hline North Coast & 93 & 4.1 & 4870 & 19.1 \\
\hline Greater Southern & 123 & 5.5 & 4826 & 25.5 \\
\hline Greater Western & 76 & 3.4 & 4002 & 19.0 \\
\hline ACT & 118 & 5.2 & 4120 & 28.6 \\
\hline Overseas & 6 & 0.3 & 0 & 0.0 \\
\hline Interstate & 13 & 0.6 & 770 & 16.9 \\
\hline Not Stated & 0 & 0.0 & 113 & 0.0 \\
\hline TOTAL & 2257 & 100.0 & 94936\# & 23.8 \\
\hline
\end{tabular}


Administration of corticosteroids to the mother prior to preterm birth improves the outcome for the infant. In 2005, 87 per cent of mothers of infants born at less than 28 weeks received corticosteroids (Figure 5, Table 69). Nearly
90 per cent of mothers of 28-31 weeks gestation infants received antenatal corticosteroids. The overall proportion of mothers receiving antenatal corticosteroids increased from 45 per cent in 1992 to 74.1 per cent in 2001.

\begin{tabular}{|c|c|c|c|c|c|c|}
\hline \multirow[t]{2}{*}{ Health Area } & \multicolumn{2}{|c|}{ Non-Aboriginal } & \multicolumn{2}{|c|}{ Aboriginal } & \multicolumn{2}{|c|}{ TOTAL } \\
\hline & No. & $\%$ & No. & $\%$ & No. & $\%$ \\
\hline Sydney South West & 423 & 98.1 & 8 & 1.9 & 431 & 20.8 \\
\hline South Eastern Sydney \& Illawarra & 265 & 98.1 & 5 & 1.9 & 270 & 13.0 \\
\hline Sydney West & 397 & 99.0 & 4 & 1.0 & 401 & 19.4 \\
\hline Northern Sydney \& Central Coast & 235 & 99.6 & 1 & 0.4 & 236 & 11.4 \\
\hline Hunter \& New England & 307 & 91.1 & 30 & 8.9 & 337 & 16.3 \\
\hline North Coast & 76 & 89.4 & 9 & 10.6 & 85 & 4.1 \\
\hline Greater Southern & 105 & 93.8 & 7 & 6.3 & 112 & 5.4 \\
\hline Greater Western & 66 & 89.2 & 8 & 10.8 & 74 & 3.6 \\
\hline ACT & 104 & 96.3 & 4 & 3.7 & 108 & 5.2 \\
\hline Interstate & 8 & 80.0 & 2 & 20.0 & 10 & 0.5 \\
\hline Overseas & 6 & 100.0 & 0 & 0.0 & 6 & 0.3 \\
\hline TOTAL & 1992 & 96.2 & 78 & 3.8 & 2070 & 100.0 \\
\hline
\end{tabular}

\section{TABLE 67}

MOTHERS OF NICUS REGISTRANTS BY HEALTH AREA OF RESIDENCE AND MATERNAL AGE, NSW \& ACT 2005

\begin{tabular}{|c|c|c|c|c|c|c|c|c|}
\hline \multirow{3}{*}{$\begin{array}{l}\text { Health Area } \\
\text { Sydney South West }\end{array}$} & \multirow{2}{*}{\multicolumn{2}{|c|}{ Less than 20}} & \multicolumn{2}{|c|}{ Maternal age (years) } & \multirow{2}{*}{\multicolumn{2}{|c|}{$35+$}} & \multirow{2}{*}{\multicolumn{2}{|c|}{ TOTAL ${ }^{\#}$}} \\
\hline & & & & & & & & \\
\hline & 15 & 3.5 & 306 & 71.0 & 110 & 25.5 & 431 & 20.9 \\
\hline South Eastern Sydney \& Illawarra & 5 & 1.9 & 186 & 69.1 & 78 & 29.0 & 269 & 13.0 \\
\hline Sydney West & 20 & 5.0 & 304 & 76.0 & 76 & 19.0 & 400 & 19.4 \\
\hline Northern Sydney \& Central Coast & 3 & 1.3 & 161 & 68.2 & 72 & 30.5 & 236 & 11.4 \\
\hline Hunter \& New England & 33 & 9.8 & 245 & 72.7 & 59 & 17.5 & 337 & 16.3 \\
\hline North Coast & 4 & 4.7 & 61 & 71.8 & 20 & 23.5 & 85 & 4.1 \\
\hline Greater Southern & 5 & 4.5 & 83 & 74.1 & 24 & 21.4 & 112 & 5.4 \\
\hline Greater Western & 4 & 5.5 & 57 & 78.1 & 12 & 16.4 & 73 & 3.5 \\
\hline ACT & 6 & 5.6 & 77 & 71.3 & 25 & 23.1 & 108 & 5.2 \\
\hline Interstate & 2 & 20.0 & 5 & 50.0 & 3 & 30.0 & 10 & 0.5 \\
\hline Overseas & 0 & 0.0 & 5 & 83.3 & 1 & 16.7 & 6 & 0.3 \\
\hline Not stated & 0 & 0.0 & 0 & 0.0 & 0 & 0.0 & 3 & 0.1 \\
\hline TOTAL & 97 & 4.7 & 1490 & 72.1 & 480 & 23.2 & 2070 & 100.0 \\
\hline
\end{tabular}

Source: NICUS Data Collection. NSW Centre for Perinatal Health Services Research

\# Total includes 3 mothers where the maternal age was not stated..

\section{TABLE 68}

MOTHERS OF NICUS REGISTRANTS BY ANTENATAL COMPLICATIONS AND GESTATIONAL AGE, NSW \& ACT 2005

Antenatal complication Gestational age (weeks)

\begin{tabular}{|c|c|c|c|c|c|c|c|c|c|c|c|c|}
\hline \multirow[t]{3}{*}{ Antenatal complication } & \multicolumn{10}{|c|}{ Gestational age (weeks) } & \multirow{2}{*}{\multicolumn{2}{|c|}{ TOTAL }} \\
\hline & \multicolumn{2}{|c|}{$22-27$} & \multicolumn{2}{|c|}{$28-31$} & \multicolumn{2}{|c|}{$32-36$} & \multicolumn{2}{|c|}{$37-41$} & \multicolumn{2}{|c|}{$42+$} & & \\
\hline & No. & $\%$ & No. & $\%$ & No. & $\%$ & No. & $\%$ & No. & $\%$ & No. & $\%$ \\
\hline Preterm labour & 166 & 76.9 & 335 & 61.8 & 392 & 53.8 & 10 & 1.7 & 0 & 0.0 & 903 & 43.6 \\
\hline Pregnancy induced hypertension & 33 & 15.3 & 113 & 20.8 & 157 & 21.6 & 46 & 8.0 & 0 & 0.0 & 349 & 16.9 \\
\hline Antepartum haemorrhage & 77 & 35.6 & 140 & 25.8 & 122 & 16.8 & 18 & 3.1 & 0 & 0.0 & 357 & 17.2 \\
\hline Intrauterine growth restriction & 20 & 9.3 & 60 & 11.1 & 93 & 12.8 & 26 & 4.5 & 0 & 0.0 & 199 & 9.6 \\
\hline Fetal distress & 29 & 13.4 & 90 & 16.6 & 119 & 16.3 & 118 & 20.5 & 0 & 0.0 & 356 & 17.2 \\
\hline Fetal diagnosis of anomaly & 1 & 0.5 & 11 & 2.0 & 58 & 8.0 & 90 & 15.6 & 0 & 0.0 & 160 & 7.7 \\
\hline Gestational diabetes & 6 & 2.8 & 38 & 7.0 & 47 & 6.5 & 33 & 5.7 & 1 & 12.5 & 125 & 6.0 \\
\hline Chorioamnionitis & 53 & 24.4 & 56 & 10.3 & 40 & 5.5 & 12 & 2.1 & 0 & 0.0 & 161 & 7.8 \\
\hline Any complication & 216 & 100.0 & 542 & 100.0 & 719 & 98.8 & 326 & 56.6 & 1 & 12.5 & 1804 & 87.1 \\
\hline TOTAL MOTHERS & 216 & 100.0 & 542 & 100.0 & 728 & 100.0 & 576 & 100.0 & 8 & 100.0 & 2070 & 100.0 \\
\hline
\end{tabular}




\section{FIGURE 5}

MOTHERS OF NICUS REGISTRANTS BY ANTENATAL CORTICOSTEROID ADMINISTRATION AND GESTATIONAL AGE, NSW \& ACT 2001-2005

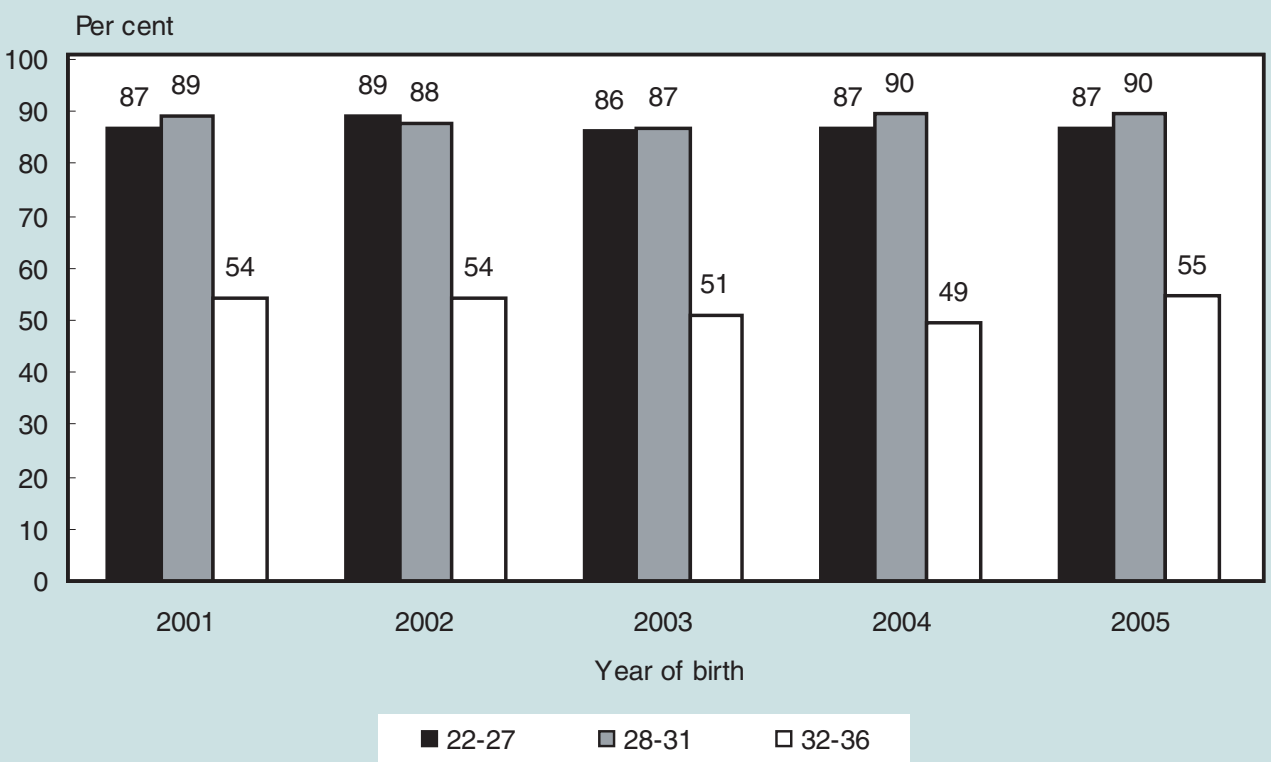

Source: NICUS Data Collection. NSW Centre for Perinatal Health Services Research.

\section{TABLE 69}

MOTHERS OF NICUS REGISTRANTS BY ANTENATAL CORTICOSTEROID ADMINISTRATION AND GESTATIONAL AGE, NSW \& ACT 2001-2005

\begin{tabular}{|c|c|c|c|c|c|c|c|c|c|}
\hline \multirow[t]{3}{*}{ Year } & \multirow{3}{*}{$\begin{array}{l}\text { Corticosteroid } \\
\text { Administration }\end{array}$} & \multicolumn{8}{|c|}{ Gestational age (weeks) } \\
\hline & & \multicolumn{2}{|c|}{$22-27$} & \multicolumn{2}{|c|}{$28-31$} & \multicolumn{2}{|c|}{$32-36$} & \multicolumn{2}{|c|}{ TOTAL } \\
\hline & & No. & $\%$ & No. & $\%$ & No. & $\%$ & No. & $\%$ \\
\hline \multirow[t]{3}{*}{2001} & No & 33 & 13.3 & 57 & 10.8 & 260 & 45.6 & 350 & 25.9 \\
\hline & Yes & 216 & 86.7 & 473 & 89.2 & 310 & 54.4 & 999 & 74.1 \\
\hline & TOTAL & 249 & 100.0 & 530 & 100.0 & 570 & 100.0 & 1349 & 100.0 \\
\hline \multirow[t]{3}{*}{2002} & No & 27 & 10.7 & 63 & 12.3 & 279 & 45.8 & 369 & 26.9 \\
\hline & Yes & 225 & 89.3 & 449 & 87.7 & 330 & 54.2 & 1004 & 73.1 \\
\hline & TOTAL & 252 & 100.0 & 512 & 100.0 & 609 & 100.0 & 1373 & 100.0 \\
\hline \multirow[t]{3}{*}{2003} & No & 31 & 13.9 & 68 & 13.1 & 311 & 49.1 & 410 & 29.8 \\
\hline & Yes & 192 & 86.1 & 451 & 86.9 & 322 & 50.9 & 965 & 70.2 \\
\hline & TOTAL & 223 & 100.0 & 519 & 100.0 & 633 & 100.0 & 1375 & 100.0 \\
\hline \multirow[t]{3}{*}{2004} & No & 30 & 13.2 & 56 & 10.3 & 361 & 50.7 & 447 & 30.1 \\
\hline & Yes & 197 & 86.8 & 490 & 89.7 & 351 & 49.3 & 1038 & 69.9 \\
\hline & TOTAL & 227 & 100.0 & 546 & 100.0 & 712 & 100.0 & 1485 & 100.0 \\
\hline \multirow[t]{3}{*}{2005} & No & 28 & 13.0 & 57 & 10.5 & 328 & 45.1 & 413 & 27.8 \\
\hline & Yes & 188 & 87.0 & 485 & 89.5 & 400 & 54.9 & 1073 & 72.2 \\
\hline & TOTAL & 216 & 100.0 & 542 & 100.0 & 728 & 100.0 & 1486 & 100.0 \\
\hline
\end{tabular}




\section{Transfer status, labour and delivery}

Infants are admitted to a neonatal intensive care unit after:

- delivery that has been booked to occur in a tertiary centre;

- delivery in a tertiary centre following maternal transfer;

- delivery in a non-tertiary centre followed by infant transfer to a tertiary centre.

Thirty-six per cent of all births were booked at a tertiary centre, ranging from 36.2 per cent for the 22-27 week gestational age group to 39.6 per cent for the 32-36 weeks gestational age group (Table 70). Maternal transfer was most common at gestations less than 32 weeks. The rate of maternal transfer was similar for infants born before 28 weeks gestation (52.7 per cent) and for those born at 28-31 weeks gestation (56.1 per cent). The overall rate of maternal transfer was 33.1 per cent.

Nearly 30 per cent of infants were transferred to a tertiary centre following birth. There were 4.4 per cent $(73 / 2,257)$ of infants transferred from one tertiary centre to another during the first day of life for assisted ventilation and/or major surgery. Transfer following birth was most common in the 37-plus weeks gestational age group (60.1 per cent). Forty-seven infants $(47 / 1,362 ; 3.5$ per cent) greater than 31 weeks gestation were discharged home prior to the admission that qualified them for registration in NICUS.

The inverse relationship between gestational age groups and the proportion of births in a tertiary centre is shown in Figure 6 and Table 71. The proportion of infants born in a tertiary centre increased from 60 per cent in 1992 to 74.8 per cent 2000. In 2005, 88.9 per cent of infants less than 32 weeks gestation were born in a tertiary centre compared with 71.2 per cent of 32-36 week gestation infants and 50 per cent of term infants.

The pattern of transfer status (Table 72) and place of birth by birth weight (Table 73) is similar to that of gestational age, with the majority ( 89 per cent) of the very low birth weight infants (less than 1,500 grams) born in a tertiary centre.

Spontaneous onset of labour was more common among mothers of infants less than 28 weeks gestation (Table 74). Augmentation and induction of labour were most common in term and post-term births. Similarly spontaneous onset of labour occurred in the majority (60.1 per cent) of all mothers of infants less than 2,500 grams birthweight (Table 75). As expected, augmentation, or induction of labour was most common in mothers of infants with a birthweight of 2,500 grams or more (27.7 per cent).

Prolonged rupture of membranes (greater than 24 hours) was more common at lower gestations, affecting 24.7 per cent of infants less than 28 weeks gestation (Table 76).

The proportion of mothers who gave birth by elective caesarean section (caesarean section without labour) was 40.3 per cent in 2005, previously ranging from 27 per cent in 1992 to 38.4 per cent in 2003 (Tables 77 and 78). The most common type of delivery was caesarean section (58.5 per cent in 1993 to 59.7 per cent in 2004), followed by 33.7 per cent for normal vaginal delivery (previously 41.9 per cent in 1993 to 35.6 per cent in 2003) and 3.2 per cent for vaginal breech delivery ( 7 per cent in 1998 to 4.2 per cent in 2003). The high rate of caesarean section and breech delivery in the NICUS cohort is related to the high proportion of preterm births. The rate of caesarean section in term and post-term births was 44.3 per cent, compared with 30.3 per cent for all livebirths in NSW and the ACT in 2005.

Continued on page 62

\section{TABLE 70}

NICUS REGISTRANTS BY BOOKING STATUS, TRANSFER STATUS AND GESTATIONAL AGE, NSW \& ACT 2005

\begin{tabular}{|c|c|c|c|c|c|c|c|c|c|c|c|c|}
\hline \multirow{3}{*}{$\begin{array}{l}\text { Booking status and } \\
\text { transfer status }\end{array}$} & \multicolumn{12}{|c|}{ Gestational age (weeks) } \\
\hline & \multicolumn{2}{|c|}{$22-27$} & \multicolumn{2}{|c|}{ 28-31 } & \multicolumn{2}{|c|}{$32-36$} & \multicolumn{2}{|c|}{$37-41$} & \multicolumn{2}{|c|}{$42+$} & \multicolumn{2}{|c|}{ TOTAL } \\
\hline & No. & $\%$ & No. & $\%$ & No. & $\%$ & No. & $\%$ & No. & $\%$ & No. & $\%$ \\
\hline Booked at tertiary hosp & 88 & 36.2 & 215 & 33.0 & 308 & 39.6 & 184 & 31.9 & 6 & 75.0 & 801 & 35.5 \\
\hline Transfer before birth & 128 & 52.7 & 366 & 56.1 & 227 & 29.2 & 26 & 4.5 & 0 & 0.0 & 747 & 33.1 \\
\hline Transfer after birth & 27 & 11.1 & 67 & 10.3 & 220 & 28.3 & 349 & 60.6 & 2 & 25.0 & 665 & 29.5 \\
\hline Booked at non tertiary hosp & 0 & 0.0 & 4 & 0.6 & 23 & 3.0 & 17 & 3.0 & 0 & 0.0 & 44 & 1.9 \\
\hline TOTAL & 243 & 100.0 & 652 & 100.0 & 778 & 100.0 & 576 & 100.0 & 8 & 100.0 & 2257 & 100.0 \\
\hline
\end{tabular}

Source: NICUS Data Collection. NSW Centre for Perinatal Health Services Research. 


\section{FIGURE 6}

Per cent

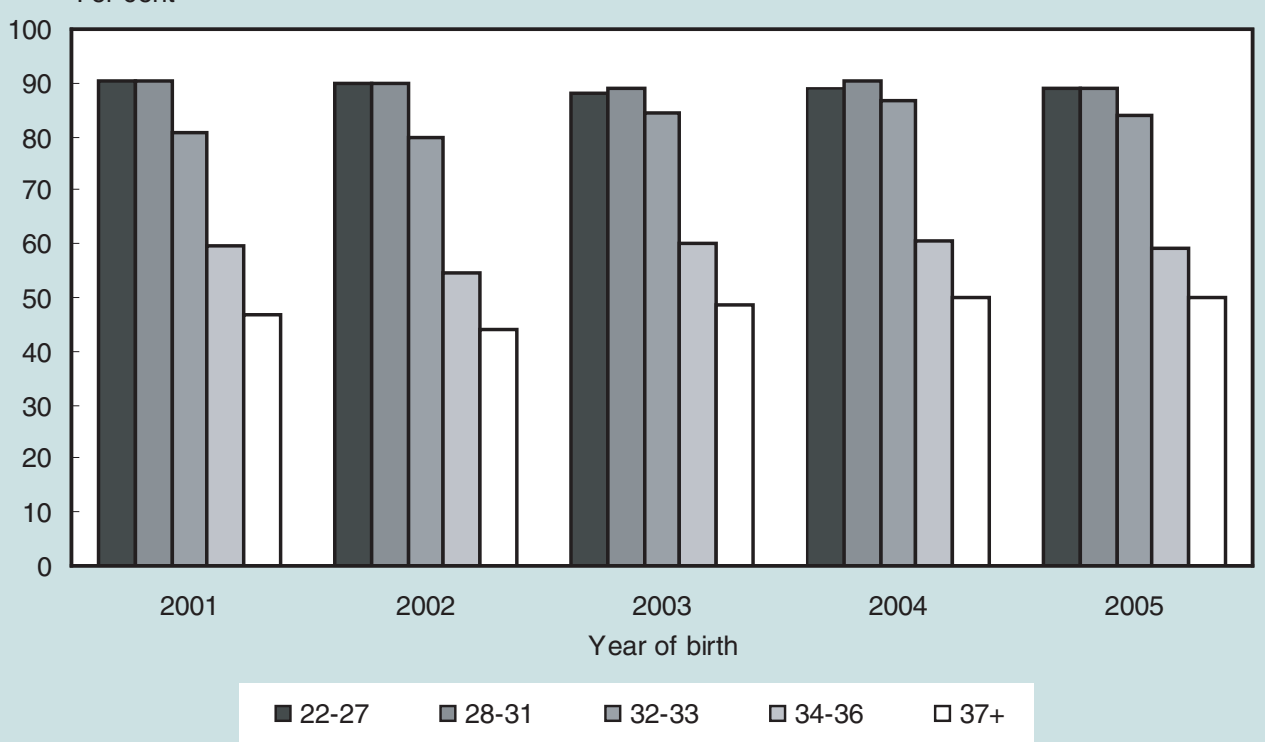

Source: NICUS Data Collection. NSW Centre for Perinatal Health Services Research.

\section{TABLE 71}

NICUS REGISTRANTS BY PLACE OF BIRTH (LEVEL OF OBSTETRIC HOSPITAL) AND GESTATIONAL AGE, NSW \& ACT 2005

\begin{tabular}{|c|c|c|c|c|c|c|c|c|c|c|c|c|}
\hline \multirow[t]{3}{*}{ Place of birth } & \multicolumn{12}{|c|}{ Gestational age (weeks) } \\
\hline & \multicolumn{2}{|c|}{ 22-27 } & \multicolumn{2}{|c|}{ 28-31 } & \multicolumn{2}{|c|}{$32-33$} & \multicolumn{2}{|c|}{$34-36$} & \multicolumn{2}{|c|}{$37+$} & \multicolumn{2}{|c|}{ TOTAL } \\
\hline & No. & $\%$ & No. & $\%$ & No. & $\%$ & No. & $\%$ & No. & $\%$ & No. & $\%$ \\
\hline Level 1 & 1 & 0.4 & 0 & 0.0 & 2 & 0.5 & 0 & 0.0 & 1 & 0.2 & 4 & 0.2 \\
\hline Level 2 & 0 & 0.0 & 3 & 0.5 & 1 & 0.3 & 3 & 0.8 & 9 & 1.5 & 16 & 0.7 \\
\hline Level 3 & 0 & 0.0 & 11 & 1.7 & 7 & 1.8 & 10 & 2.5 & 29 & 5.0 & 57 & 2.5 \\
\hline Level 4 & 8 & 3.3 & 24 & 3.7 & 14 & 3.7 & 39 & 9.9 & 92 & 15.8 & 177 & 7.8 \\
\hline Level 5 & 10 & 4.1 & 23 & 3.5 & 20 & 5.2 & 40 & 10.1 & 68 & 11.6 & 161 & 7.1 \\
\hline Level 6 & 216 & 88.9 & $580 \#$ & 89.0 & 321 & 83.8 & 233 & 59.0 & 292 & 50.0 & 1642 & 72.8 \\
\hline Private hospital & 3 & 1.2 & 9 & 1.4 & 13 & 3.4 & 64 & 16.2 & 80 & 13.7 & 169 & 7.5 \\
\hline Born before arrival & 0 & 0.0 & 2 & 0.3 & 3 & 0.8 & 2 & 0.5 & 2 & 0.3 & 9 & 0.4 \\
\hline Interstate-Overseas & 5 & 2.1 & 0 & 0.0 & 2 & 0.5 & 4 & 1.0 & 11 & 2.0 & 22 & 1.0 \\
\hline TOTAL & 243 & 100.0 & 652 & 100.0 & 383 & 100.0 & 395 & 100.0 & 584 & 100.0 & 2257 & 100.0 \\
\hline
\end{tabular}

Source: NICUS Data Collection. NSW Centre for Perinatal Health Services Research.

\# 368/580 (63.4\%) babies not born in a level 6 hospital were 30-31 weeks gestation.

\section{TABLE 72}

NICUS REGISTRANTS BY BOOKING STATUS, TRANSFER STATUS AND BIRTH WEIGHT, NSW \& ACT 2005

$\begin{array}{lll}\text { Booking status and Birth weight (grams) } & \text { B }\end{array}$

\begin{tabular}{|c|c|c|c|c|c|c|c|c|c|c|}
\hline transfer status & \multicolumn{2}{|c|}{ Less than 1,000} & \multicolumn{2}{|c|}{$1,000-1,499$} & \multicolumn{2}{|c|}{$1,500-2,499$} & \multicolumn{2}{|c|}{$2,500+$} & \multicolumn{2}{|c|}{ TOTAL } \\
\hline Booked at tertiary hosp & 92 & 36.5 & 164 & 34.3 & 281 & 36.9 & 264 & 34.5 & 801 & 35.5 \\
\hline Transfer before birth & 137 & 54.4 & 258 & 54.0 & 289 & 37.9 & 63 & 8.2 & 747 & 33.1 \\
\hline Booked at non tertiary hosp & 0 & 0.0 & 5 & 1.0 & 15 & 2.0 & 24 & 3.1 & 44 & 1.9 \\
\hline TOTAL & 252 & 100.0 & 478 & 100.0 & 762 & 100.0 & 765 & 100.0 & 2257 & 100.0 \\
\hline
\end{tabular}

Source: NICUS Data Collection. NSW Centre for Perinatal Health Services Research. 


\section{TABLE 73}

NICUS REGISTRANTS BY PLACE OF BIRTH (LEVEL OF OBSTETRIC HOSPITAL) AND BIRTH WEIGHT, NSW \& ACT 2005

Place of birth Birth weight (grams)

\begin{tabular}{|c|c|c|c|c|c|c|c|c|c|c|}
\hline \multirow[t]{3}{*}{ Place of birth } & \multicolumn{10}{|c|}{ Birth weight (grams) } \\
\hline & \multicolumn{2}{|c|}{$<1,000$} & \multicolumn{2}{|c|}{$1,000-1,499$} & \multicolumn{2}{|c|}{$1,500-2,499$} & \multicolumn{2}{|c|}{$2,500+$} & \multicolumn{2}{|c|}{ TOTAL } \\
\hline & No. & $\%$ & No. & $\%$ & No. & $\%$ & No. & $\%$ & No. & $\%$ \\
\hline Level 1 & 1 & 0.4 & 0 & 0.0 & 2 & 0.3 & 1 & 0.1 & 4 & 0.2 \\
\hline Level 2 & 0 & 0.0 & 2 & 0.4 & 5 & 0.7 & 9 & 1.2 & 16 & 0.7 \\
\hline Level 3 & 0 & 0.0 & 7 & 1.5 & 20 & 2.6 & 30 & 3.9 & 57 & 2.5 \\
\hline Level 4 & 5 & 2.0 & 18 & 3.8 & 49 & 6.4 & 105 & 13.7 & 177 & 7.8 \\
\hline Level 5 & 11 & 4.4 & 18 & 3.8 & 51 & 6.7 & 81 & 10.6 & 161 & 7.1 \\
\hline Level 6 & 229 & 90.9 & 421 & 88.1 & 588 & 77.2 & 404 & 52.8 & 1642 & 72.8 \\
\hline Private hospital & 2 & 0.8 & 10 & 2.1 & 37 & 4.9 & 120 & 15.7 & 169 & 7.5 \\
\hline Born before arrival & 1 & 0.4 & 0 & 0.0 & 6 & 0.8 & 2 & 0.3 & 9 & 0.4 \\
\hline Interstate-Overseas & 3 & 1.2 & 2 & 0.4 & 4 & 0.5 & 13 & 1.7 & 22 & 1.0 \\
\hline TOTAL & 252 & 100.0 & 478 & 100.0 & 762 & 100.0 & 765 & 100.0 & 2257 & 100.0 \\
\hline
\end{tabular}

\section{TABLE 74}

MOTHERS OF NICUS REGISTRANTS BY ONSET OF LABOUR AND GESTATIONAL AGE, NSW \& ACT 2005

\begin{tabular}{|c|c|c|c|c|c|c|c|c|c|c|c|c|}
\hline \multirow{3}{*}{ Onset of labour } & \multicolumn{12}{|c|}{ Gestational age (weeks) } \\
\hline & \multicolumn{2}{|c|}{$22-27$} & \multicolumn{2}{|c|}{$28-31$} & \multicolumn{2}{|c|}{$32-36$} & \multicolumn{2}{|c|}{$37-41$} & \multicolumn{2}{|c|}{$42+$} & \multicolumn{2}{|c|}{ TOTAL } \\
\hline & No. & $\%$ & No. & $\%$ & No. & $\%$ & No. & $\%$ & No. & $\%$ & No. & $\%$ \\
\hline Spontaneous & 134 & 62.0 & 270 & 49.8 & 318 & 43.7 & 230 & 39.9 & 4 & 50.0 & 956 & 46.2 \\
\hline Augmented & 12 & 5.6 & 19 & 3.5 & 26 & 3.6 & 46 & 8.0 & 0 & 0.0 & 103 & 5.0 \\
\hline Induced & 3 & 1.4 & 3 & 0.6 & 47 & 6.5 & 143 & 24.8 & 4 & 50.0 & 200 & 9.7 \\
\hline No labour & 67 & 31.0 & 250 & 46.1 & 337 & 46.3 & 157 & 27.3 & 0 & 0.0 & 811 & 39.2 \\
\hline TOTAL & 216 & 100.0 & 542 & 100.0 & 728 & 100.0 & 576 & 100.0 & 8 & 100.0 & 2070 & 100.0 \\
\hline
\end{tabular}

\section{TABLE 75}

MOTHERS OF NICUS REGISTRANTS BY ONSET OF LABOUR AND BIRTH WEIGHT, NSW \& ACT 2005

Onset of labour Birth weight (grams)

\begin{tabular}{|c|c|c|c|c|c|c|c|c|c|c|}
\hline \multirow[t]{2}{*}{ - } & \multicolumn{2}{|c|}{ Less than 1,000} & \multicolumn{2}{|c|}{$1,000-1,499$} & \multicolumn{2}{|c|}{$1,500-2,499$} & \multicolumn{2}{|c|}{$2,500+$} & \multicolumn{2}{|c|}{ TOTAL } \\
\hline & No. & $\%$ & No. & $\%$ & No. & $\%$ & No. & $\%$ & No. & $\%$ \\
\hline Spontaneous & 111 & 49.8 & 181 & 44.6 & 361 & 53.0 & 303 & 39.9 & 956 & 46.2 \\
\hline Augmented & 10 & 4.5 & 12 & 3.0 & 31 & 4.5 & 50 & 6.6 & 103 & 5.0 \\
\hline Induced & 2 & 0.9 & 6 & 1.5 & 32 & 4.7 & 160 & 21.1 & 200 & 9.7 \\
\hline No labour & 100 & 44.8 & 207 & 51.0 & 257 & 37.7 & 247 & 32.5 & 811 & 39.2 \\
\hline TOTAL & 223 & 100.0 & 406 & 100.0 & 681 & 100.0 & 760 & 100.0 & 2070 & 100.0 \\
\hline
\end{tabular}

\section{TABLE 76}

NICUS REGISTRANTS BY DURATION OF RUPTURE OF MEMBRANES AND GESTATIONAL AGE, NSW \& ACT 2005

\begin{tabular}{|c|c|c|c|c|c|c|c|c|c|c|c|c|}
\hline \multirow{3}{*}{$\begin{array}{l}\text { Duration of rupture of } \\
\text { membranes }\end{array}$} & \multicolumn{12}{|c|}{ Gestational age (weeks) } \\
\hline & & $2-27$ & & $8-31$ & & $2-36$ & & & & 2+ & & TAL \\
\hline & No. & $\%$ & No. & $\%$ & No. & $\%$ & No. & $\%$ & No. & $\%$ & No. & $\%$ \\
\hline Less than 24 hours & 183 & 75.3 & 519 & 79.6 & 694 & 89.2 & 554 & 96.2 & 8 & 100.0 & 1958 & \\
\hline 24 hours -7 days & 35 & 14.4 & 70 & 10.7 & 45 & 5.8 & 20 & 3.5 & 0 & 0.0 & 170 & \\
\hline $8+$ days & 25 & 10.3 & 63 & 9.7 & 39 & 5.0 & 2 & 0.3 & 0 & 0.0 & 129 & 5. \\
\hline TOTAL & 243 & 100.0 & 652 & 100.0 & 778 & 100.0 & 576 & 100.0 & 8 & 100.0 & 2257 & 100. \\
\hline
\end{tabular}

Source: NICUS Data Collection. NSW Centre for Perinatal Health Services Research. 
TABLE 77

NICUS REGISTRANTS BY TYPE OF DELIVERY AND GESTATIONAL AGE, NSW \& ACT 2005

\begin{tabular}{|c|c|c|c|c|c|c|c|c|c|c|c|c|}
\hline \multirow[t]{3}{*}{ Type of delivery } & \multicolumn{12}{|c|}{ Gestational age (weeks) } \\
\hline & \multicolumn{2}{|c|}{ 22-27 } & \multicolumn{2}{|c|}{ 28-31 } & \multicolumn{2}{|c|}{$32-36$} & \multicolumn{2}{|c|}{$37-41$} & \multicolumn{2}{|c|}{$42+$} & \multicolumn{2}{|c|}{ TOTAL } \\
\hline & No. & $\%$ & No. & $\%$ & No. & $\%$ & No. & $\%$ & No. & $\%$ & No. & $\%$ \\
\hline Normal vaginal & 92 & 37.9 & 190 & 29.1 & 212 & 27.2 & 262 & 45.5 & 4 & 50.0 & 760 & 33.7 \\
\hline Forceps & 7 & 2.9 & 9 & 1.4 & 14 & 1.8 & 19 & 3.3 & 0 & 0.0 & 49 & 2.2 \\
\hline Forceps rotation & 0 & 0.0 & 2 & 0.3 & 0 & 0.0 & 2 & 0.3 & 0 & 0.0 & 4 & 0.2 \\
\hline Vacuum extraction & 1 & 0.4 & 1 & 0.2 & 13 & 1.7 & 33 & 5.7 & 3 & 37.5 & 51 & 2.3 \\
\hline Vaginal breech & 20 & 8.2 & 30 & 4.6 & 20 & 2.6 & 2 & 0.3 & 0 & 0.0 & 72 & 3.2 \\
\hline Elective Caesarean & 75 & 30.9 & 296 & 45.4 & 379 & 48.7 & 160 & 27.8 & 0 & 0.0 & 910 & 40.3 \\
\hline Emergency Caesarean & 48 & 19.8 & 124 & 19.0 & 140 & 18.0 & 98 & 17.0 & 1 & 12.5 & 411 & 18.2 \\
\hline TOTAL & 243 & 100.0 & 652 & 100.0 & 778 & 100.0 & 576 & 100.0 & 8 & 100.0 & 2257 & 100.0 \\
\hline
\end{tabular}

\section{TABLE 78}

NICUS REGISTRANTS BY TYPE OF DELIVERY AND BIRTH WEIGHT, NSW \& ACT 2005

\begin{tabular}{|c|c|c|c|c|c|c|c|c|c|c|}
\hline \multirow[t]{3}{*}{ Type of delivery } & \multicolumn{10}{|c|}{ Birth weight (grams) } \\
\hline & \multicolumn{2}{|c|}{ Less than 1,000} & \multicolumn{2}{|c|}{$1,000-1,499$} & \multicolumn{2}{|c|}{$1,500-2,499$} & \multicolumn{2}{|c|}{$2,500+$} & \multicolumn{2}{|c|}{ TOTAL } \\
\hline & No. & $\%$ & No. & $\%$ & No. & $\%$ & No. & $\%$ & No. & $\%$ \\
\hline Normal vaginal & 72 & 28.6 & 115 & 24.1 & 266 & 34.9 & 307 & 40.1 & 760 & 33.7 \\
\hline Forceps & 6 & 2.4 & 4 & 0.8 & 13 & 1.7 & 26 & 3.4 & 49 & 2.2 \\
\hline Forceps rotation & 0 & 0.0 & 0 & 0.0 & 2 & 0.3 & 2 & 0.3 & 4 & 0.2 \\
\hline Vacuum extraction & 1 & 0.4 & 1 & 0.2 & 5 & 0.7 & 44 & 5.8 & 51 & 2.3 \\
\hline Vaginal breech & 19 & 7.5 & 20 & 4.2 & 28 & 3.7 & 5 & 0.7 & 72 & 3.2 \\
\hline Elective Caesarean & 113 & 44.8 & 247 & 51.7 & 292 & 38.3 & 258 & 33.7 & 910 & 40.3 \\
\hline Emergency Caesarean & 41 & 16.3 & 91 & 19.0 & 156 & 20.5 & 123 & 16.1 & 411 & 18.2 \\
\hline TOTAL & 252 & 100.0 & 478 & 100.0 & 762 & 100.0 & 765 & 100.0 & 2257 & 100.0 \\
\hline
\end{tabular}

Source: NICUS Data Collection. NSW Centre for Perinatal Health Services Research.

\section{(Continued from page 59)}

\section{Infant characteristics}

Three quarters of the infants (74.1 per cent) were preterm (less than 37 weeks gestation), 39.7 per cent were very preterm (less than 32 weeks gestation) and 10.8 per cent were extremely preterm (less than 28 weeks gestation) (Figure 7). The proportion of infants in each gestational age group has remained relatively constant (Table 79). Almost all liveborn infants at 25-31 weeks gestation were admitted to a NICU, nearly two-thirds at 32 weeks gestation, and one-fifth at 33-34 weeks gestation (Table 80).

Sixty-six per cent of infants had a low birth weight (less than 2,500 grams), 32.3 per cent had a very low birth weight (less than 1,500 grams) and 11.2 per cent had an extremely low birth weight (less than 1,000 grams). The proportion of infants in each birth weight group has remained relatively constant (Table 81). Almost all liveborn infants 600-1500 grams birth weight were admitted to a NICU (Table 82).

Overall, 58.2 per cent of infants were male. The ratio of males to females remains at 3:2 in most gestational age groups (Table 83).

The overall proportion of the infants who had a major congenital anomaly decreased from 22 per cent in 1992 to 15.6 per cent in 2005. Congenital anomalies were more common among term infants (37-plus weeks gestational age), of whom 34.8 per cent had a major congenital anomaly and 3.1 per cent had a minor congenital anomaly (Table 84).

Continued on page 66 


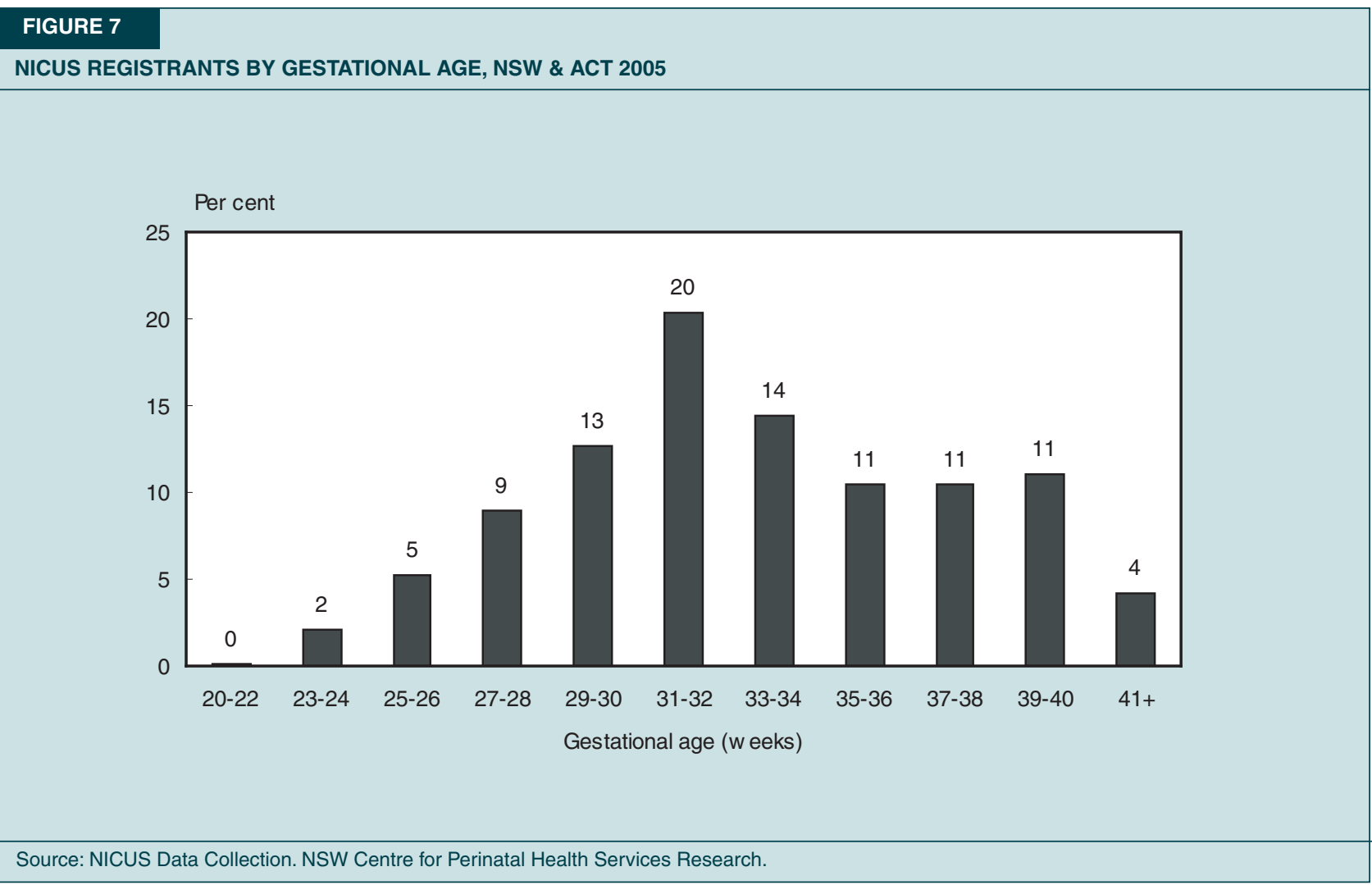

\begin{tabular}{|c|c|c|c|c|c|c|c|c|c|c|}
\hline \multirow[t]{3}{*}{ Gestational age (weeks) } & \multicolumn{10}{|c|}{ Year of birth } \\
\hline & \multicolumn{2}{|c|}{2001} & \multicolumn{2}{|c|}{2002} & \multicolumn{2}{|c|}{2003} & \multicolumn{2}{|c|}{2004} & \multicolumn{2}{|c|}{2005} \\
\hline & No. & $\%$ & No. & $\%$ & No. & $\%$ & No. & $\%$ & No. & $\%$ \\
\hline $22-27$ & 277 & 13.8 & 282 & 14.0 & 253 & 12.0 & 264 & 11.8 & 243 & 10.8 \\
\hline 28-31 & 640 & 31.8 & 604 & 30.0 & 608 & 28.8 & 649 & 29.0 & 652 & 28.9 \\
\hline $32-36$ & 611 & 30.4 & 640 & 31.8 & 678 & 32.1 & 764 & 34.2 & 778 & 34.5 \\
\hline $37-41$ & 473 & 23.5 & 480 & 23.8 & 561 & 26.5 & 555 & 24.8 & 576 & 25.5 \\
\hline $42+$ & 9 & 0.4 & 8 & 0.4 & 14 & 0.7 & 4 & 0.2 & 8 & 0.4 \\
\hline TOTAL & 2010 & 100.0 & 2014 & 100.0 & 2114 & 100.0 & 2236 & 100.0 & 2257 & 100.0 \\
\hline
\end{tabular}


TABLE 80

BIRTHS BY NICUS REGISTRATION AND GESTATIONAL AGE, NSW \& ACT 2005

\begin{tabular}{|c|c|c|c|c|c|}
\hline $\begin{array}{l}\text { Gestational age } \\
\text { (weeks) }\end{array}$ & $\begin{array}{c}\text { NSW \& ACT NICUS } \\
\text { Stillbirths } \\
\text { No. }\end{array}$ & $\begin{array}{l}\text { Live births } \\
\text { No. }\end{array}$ & $\begin{array}{c}\text { Registrations } \\
\text { No. }\end{array}$ & $\begin{array}{l}\text { Rate per } 1,000 \\
\text { live births }\end{array}$ & $\begin{array}{c}\% \\
\text { of cohort }\end{array}$ \\
\hline Less than 21 & 62 & 11 & 0 & 0.0 & 0.0 \\
\hline 21 & 69 & 26 & 0 & 0.0 & 0.0 \\
\hline 22 & 48 & 40 & 1 & 25.0 & 0.1 \\
\hline 23 & 31 & 39 & 7 & 179.5 & 0.3 \\
\hline 24 & 29 & 60 & 40 & 666.7 & 1.8 \\
\hline 25 & 25 & 55 & 53 & 963.6 & 2.4 \\
\hline 26 & 29 & 45 & 65 & 1444.4 & 2.9 \\
\hline 27 & 14 & 86 & 77 & 895.3 & 3.4 \\
\hline 28 & 12 & 124 & 123 & 991.9 & 5.5 \\
\hline 29 & 13 & 117 & 117 & 1000.0 & 5.2 \\
\hline 30 & 11 & 168 & 169 & 1006.0 & 7.5 \\
\hline 31 & 18 & 247 & 243 & 983.8 & 10.8 \\
\hline 32 & 16 & 369 & 215 & 582.7 & 9.5 \\
\hline 33 & 13 & 486 & 168 & 345.7 & 7.4 \\
\hline 34 & 19 & 889 & 158 & 177.7 & 7.0 \\
\hline 35 & 14 & 1359 & 122 & 89.8 & 5.4 \\
\hline 36 & 17 & 2448 & 115 & 47.0 & 5.1 \\
\hline 37 & 20 & 5471 & 95 & 17.4 & 4.2 \\
\hline 38 & 24 & 15377 & 143 & 9.3 & 6.3 \\
\hline 39 & 30 & 23044 & 120 & 5.2 & 5.3 \\
\hline 40 & 30 & 27150 & 131 & 4.8 & 5.8 \\
\hline 41 & 21 & 15535 & 87 & 5.6 & 3.9 \\
\hline 42 & 2 & 1640 & 8 & 4.9 & 0.4 \\
\hline 43 & 0 & 135 & 0 & 0.0 & 0.0 \\
\hline 44 & 0 & 3 & 0 & 0.0 & 0.0 \\
\hline 45 & 0 & 1 & 0 & 0.0 & 0.0 \\
\hline 46 & 0 & 1 & 0 & 0.0 & 0.0 \\
\hline Not stated & 1 & 10 & 0 & 0.0 & 0.0 \\
\hline TOTAL & 568 & 94936\# & 2257 & 23.8 & 100.0 \\
\hline
\end{tabular}

\section{TABLE 81}

NICUS REGISTRANTS BY BIRTH WEIGHT, NSW \& ACT 2001-2005

\begin{tabular}{|c|c|c|c|c|c|c|c|c|c|c|}
\hline \multirow[t]{3}{*}{ Birth weight (grams) } & \multicolumn{10}{|c|}{ Year of birth } \\
\hline & \multicolumn{2}{|c|}{2001} & \multicolumn{2}{|c|}{2002} & \multicolumn{2}{|c|}{2003} & \multicolumn{2}{|c|}{2004} & \multicolumn{2}{|c|}{2005} \\
\hline & No. & $\%$ & No. & $\%$ & No. & $\%$ & No. & $\%$ & No. & $\%$ \\
\hline Less than 400 & 2 & 0.1 & 2 & 0.1 & 2 & 0.1 & 1 & 0.0 & 0 & 0.0 \\
\hline $400-499$ & 5 & 0.2 & 7 & 0.3 & 9 & 0.4 & 5 & 0.2 & 5 & 0.2 \\
\hline $500-599$ & 30 & 1.5 & 21 & 1.0 & 24 & 1.1 & 25 & 1.1 & 19 & 0.8 \\
\hline $600-699$ & 49 & 2.4 & 53 & 2.6 & 38 & 1.8 & 35 & 1.6 & 51 & 2.3 \\
\hline $700-799$ & 49 & 2.4 & 63 & 3.1 & 54 & 2.6 & 54 & 2.4 & 53 & 2.3 \\
\hline $800-899$ & 72 & 3.6 & 58 & 2.9 & 60 & 2.8 & 66 & 3.0 & 54 & 2.4 \\
\hline 900-999 & 63 & 3.1 & 81 & 4.0 & 80 & 3.8 & 77 & 3.4 & 70 & 3.1 \\
\hline $1,000-1,249$ & 219 & 10.9 & 181 & 9.0 & 197 & 9.3 & 232 & 10.4 & 233 & 10.3 \\
\hline $1,250-1,499$ & 274 & 13.6 & 264 & 13.1 & 257 & 12.2 & 279 & 12.5 & 245 & 10.9 \\
\hline $1,500-1,749$ & 231 & 11.5 & 228 & 11.3 & 215 & 10.2 & 257 & 11.5 & 244 & 10.8 \\
\hline $1,750-1,999$ & 159 & 7.9 & 163 & 8.1 & 185 & 8.8 & 174 & 7.8 & 211 & 9.3 \\
\hline $2,000-2,499$ & 251 & 12.5 & 273 & 13.6 & 258 & 12.2 & 280 & 12.5 & 307 & 13.6 \\
\hline $2,500-2,999$ & 215 & 10.7 & 205 & 10.2 & 244 & 11.5 & 280 & 12.5 & 249 & 11.0 \\
\hline $3,000-3,499$ & 195 & 9.7 & 195 & 9.7 & 228 & 10.8 & 227 & 10.2 & 277 & 12.3 \\
\hline $3,500-3,999$ & 132 & 6.6 & 158 & 7.8 & 176 & 8.3 & 153 & 6.8 & 147 & 6.5 \\
\hline $4,000+$ & 64 & 3.2 & 62 & 3.1 & 87 & 4.1 & 91 & 4.1 & 92 & 4.1 \\
\hline TOTAL & 2010 & 100.0 & 2014 & 100.0 & 2114 & 100.0 & 2236 & 100.0 & 2257 & 100.0 \\
\hline
\end{tabular}


TABLE 82

BIRTHS BY NICUS REGISTRATION AND BIRTH WEIGHT, NSW \& ACT 2005

\begin{tabular}{lcccc}
$\begin{array}{l}\text { Birth weight } \\
\text { (grams) }\end{array}$ & \multicolumn{2}{c}{ NSW \& ACT } & \multicolumn{2}{c}{ NICUS } \\
& $\begin{array}{c}\text { Stillbirths } \\
\text { No. }\end{array}$ & $\begin{array}{c}\text { Live births } \\
\text { No. }\end{array}$ & $\begin{array}{c}\text { Registrations } \\
\text { No. }\end{array}$ & $\begin{array}{c}\text { Rate per } 1,000 \\
\text { live births }\end{array}$ \\
of cohort
\end{tabular}

Less than 400

400-499

500-599

600-699

700-799

800-899

900-999

$\begin{array}{rr}151 & 53 \\ 61 & 41 \\ 37 & 61 \\ 34 & 69 \\ 18 & 55 \\ 12 & 55 \\ 15 & 68 \\ & \\ 23 & 238 \\ 19 & 275 \\ 13 & 462 \\ 19 & 704 \\ & \\ 49 & 3631 \\ 45 & 14303 \\ 36 & 33954 \\ 25 & 29367 \\ 11 & 11600 \\ 568 & 94936 \#\end{array}$

No.

No.

live births

of cohort

$1,000-1,249$

$1,250-1,499$

$1,500-1,749$

$1,750-1,999$

2,000-2,499

$2,500-2,999$

$3,000-3,499$

3,500-3,999

$4,000+$

TOTAL

53
41
61

61

$69-51$

55

$55-54$

$68-70$

0.0
122.0

122.0

311.5

739.1
963.6

$\begin{array}{rr}53 & 963.6 \\ 54 & 981.8\end{array}$

1029.4

0.0

0.2
0.8

2.3

38

1029.4

2.4

75

528.1

299.7

10.9

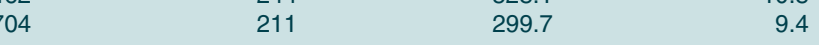

Source: NICUS Data Collection. NSW Centre for Perinatal Health Services Research. NSW Midwives Data Collection 2005. Centre for Epidemiology and Research, NSW Department of Health ACT Maternal Perinatal Data Collection 2004, ACT Health.

\# Excludes 32 babies reported to the MDC in 2005 for whom the birth outcome was not known.

\section{TABLE 83}

NICUS REGISTRANTS BY GENDER AND GESTATIONAL AGE, NSW \& ACT 2005

Sex Gestational age (weeks)

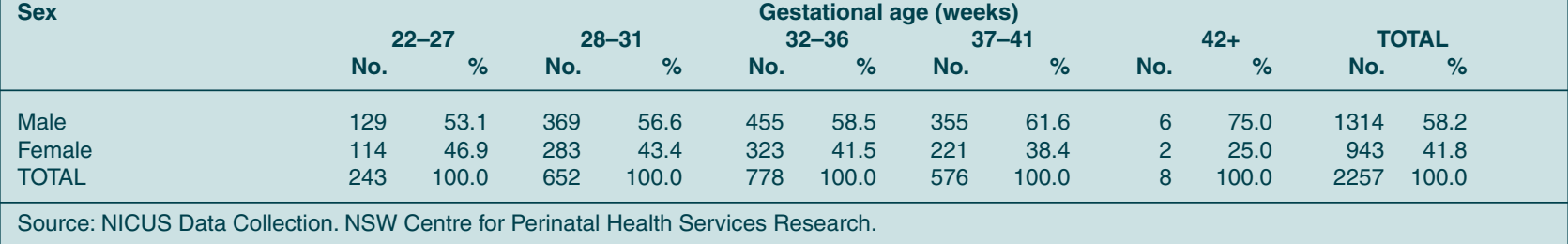

\section{TABLE 84}

NICUS REGISTRANTS BY CONGENITAL ANOMALIES AND GESTATIONAL AGE, NSW \& ACT 2005

Congenital anomaly Gestational age (weeks)

\begin{tabular}{|c|c|c|c|c|c|c|c|c|c|c|c|c|}
\hline \multirow[t]{3}{*}{ Congenital anomaly } & \multicolumn{12}{|c|}{ Gestational age (weeks) } \\
\hline & \multicolumn{2}{|c|}{$22-27$} & \multicolumn{2}{|c|}{ 28-31 } & \multicolumn{2}{|c|}{$32-36$} & \multicolumn{2}{|c|}{$37-41$} & \multicolumn{2}{|c|}{$42+$} & \multicolumn{2}{|c|}{ TOTAL } \\
\hline & No. & $\%$ & No. & $\%$ & No. & $\%$ & No. & $\%$ & No. & $\%$ & No. & $\%$ \\
\hline None & 222 & 91.4 & 614 & 94.2 & 665 & 85.5 & 356 & 61.8 & 7 & 87.5 & 1864 & 82.6 \\
\hline Minor & 4 & 1.6 & 6 & 0.9 & 13 & 1.7 & 18 & 3.1 & 0 & 0.0 & 41 & 1.8 \\
\hline Major & 17 & 7.0 & 32 & 4.9 & 100 & 12.9 & 202 & 35.1 & 1 & 12.5 & 352 & 15.6 \\
\hline TOTAL & 243 & 100.0 & 652 & 100.0 & 778 & 100.0 & 576 & 100.0 & 8 & 100.0 & 2257 & 100.0 \\
\hline
\end{tabular}




\section{(Continued from page 62)}

The overall proportion of infants born following a multiple pregnancy was 21 per cent in 2005 (range 14.5 per cent in 1993 to 22.4 per cent in 2001). In 2005, most of the infants (79 per cent) were from a singleton pregnancy, 18.3 per cent were from a twin pregnancy, 2.3 per cent were from a triplet pregnancy and 0.3 per cent were from a quadruplet pregnancy. The higher than expected rate of multiple births among the 2005 NICUS cohort reflects the high proportion of multiple pregnancies resulting in preterm birth with 20.2 per cent of infants less than 37 weeks gestation (Table 85). Multiple births represented 3.3 per cent of all NSW/ACT livebirths in 2005.

Table 86 shows the median, 25th and 75th percentiles for one- and five-minute Apgar scores according to gestational age groups. For infants 28-42 weeks gestational age, the median one-minute Apgar score was seven. The median five-minute score was nine for infants 28-42 weeks gestational age. The proportion of infants with a one-minute Apgar score of $0-4$ has decreased from 38.7 per cent in 1992 to 23.5 per cent in 2005 . Similarly the proportion of infants with a five-minute Apgar score of 0-4 has decreased from 10.8 per cent in 1992 to 6.2 per cent in 2005 (Table 87).

Infants with major congenital anomalies $(n=352)$ were excluded from the analysis of morbidity and mortality.

The majority of infants without a major congenital anomaly $(1,651 / 1,905 ; 86.7$ per cent) in the 2005 NICUS cohort received assisted ventilation (intermittent mandatory ventilation and/or continuous positive airways pressure) (Table 88).
The main indication for assisted ventilation for most infants was respiratory distress syndrome (Figure 8). The main indication for assisted ventilation varied with gestational age. Respiratory distress syndrome, immature lung, and transient tachypnoea were more common in the preterm groups, whereas perinatal asphyxia, meconium aspiration, pulmonary hypertension and apnoea were more common in term infants (Figure 8, Table 89).

The overall proportion of ventilated infants who received surfactant was 36.5 in 2005 (range 33.8 per cent in 1992 to 51.8 per cent in 1998) (Table 90). In 2005, 52.1 per cent of the infants who received surfactant were less than 32 weeks gestational age. Nearly half (52.4 per cent) of ventilated infants with a diagnosis of respiratory distress syndrome received surfactant.

Proven systemic infection has decreased from 21.5 per cent in 1992 to 10.4 per cent of infants in 2005. Infection was most common among infants less than 28 weeks gestation (35.8 per cent) (Table 91).

Overall, the incidence of treated patent ductus arteriosus (PDA) was 14.2 per cent in 2005 (range 10.7 in 1994 to 15.6 per cent in 2004). In 2005, 96.3 per cent of the infants treated for PDA were less than 32 weeks gestational age (Table 92). The majority of infants with a PDA requiring treatment received indomethacin only (12.6 per cent). Surgical treatment of PDA was predominantly performed on infants less than 28 weeks gestation (6.7 per cent). Some infants ( 5.8 per cent) were treated with both indomethacin and surgery.

Continued on page 70

\section{TABLE 85}

NICUS REGISTRANTS BY PLURALITY AND GESTATIONAL AGE, NSW \& ACT 2005

\begin{tabular}{|c|c|c|c|c|c|c|c|c|c|c|c|c|}
\hline \multirow[t]{3}{*}{ Plurality } & \multicolumn{12}{|c|}{ Gestational age (weeks) } \\
\hline & \multicolumn{2}{|c|}{$22-27$} & \multicolumn{2}{|c|}{$28-31$} & \multicolumn{2}{|c|}{$32-36$} & \multicolumn{2}{|c|}{$37-41$} & \multicolumn{2}{|c|}{$42+$} & \multicolumn{2}{|c|}{ TOTAL } \\
\hline & No. & $\%$ & No. & $\%$ & No. & $\%$ & No. & $\%$ & No. & $\%$ & No. & $\%$ \\
\hline Singleton & 180 & 74.1 & 438 & 67.2 & 598 & 76.9 & 560 & 97.2 & 8 & 100.0 & 1784 & 79.0 \\
\hline Twins & 55 & 22.6 & 177 & 27.1 & 166 & 21.3 & 16 & 2.8 & 0 & 0.0 & 414 & 18.3 \\
\hline Triplets & 8 & 3.3 & 33 & 5.1 & 12 & 1.5 & 0 & 0.0 & 0 & 0.0 & 53 & 2.3 \\
\hline Quads & 0 & 0.0 & 4 & 0.6 & 2 & 0.3 & 0 & 0.0 & 0 & 0.0 & 6 & 0.3 \\
\hline TOTAL & 243 & 100.0 & 652 & 100.0 & 778 & 100.0 & 576 & 100.0 & 8 & 100.0 & 2257 & 100.0 \\
\hline
\end{tabular}


TABLE 86

NICUS REGISTRANTS BY APGAR SCORE AND GESTATIONAL AGE, NSW \& ACT 2005

\begin{tabular}{|c|c|c|c|c|c|c|c|c|}
\hline \multirow[t]{2}{*}{ Apgar score } & \multicolumn{8}{|c|}{ Gestational age (weeks) } \\
\hline & \multicolumn{2}{|c|}{$\begin{array}{c}22-27 \\
\text { Median (25\%,75\%) }\end{array}$} & \multicolumn{2}{|c|}{$\begin{array}{c}28-31 \\
\text { Median (25\%,75\%) }\end{array}$} & \multicolumn{2}{|c|}{$\begin{array}{c}32-36 \\
\text { Median }(25 \%, 75 \%)\end{array}$} & \multicolumn{2}{|c|}{$\begin{array}{c}37+ \\
\text { Median }(25 \%, 75 \%)\end{array}$} \\
\hline One-minute Apgar & 5 & $(3,7)$ & 7 & $(5,8)$ & 7 & $(5,9)$ & 7 & $(5,9)$ \\
\hline Five-minute Apgar & 8 & $(6,8)$ & 9 & $(8,9)$ & 9 & $(8,9)$ & 9 & $(7,9)$ \\
\hline
\end{tabular}

\section{TABLE 87}

\section{NICUS REGISTRANTS BY APGAR SCORE AT ONE AND FIVE MINUTES, NSW \& ACT 2001-2005}

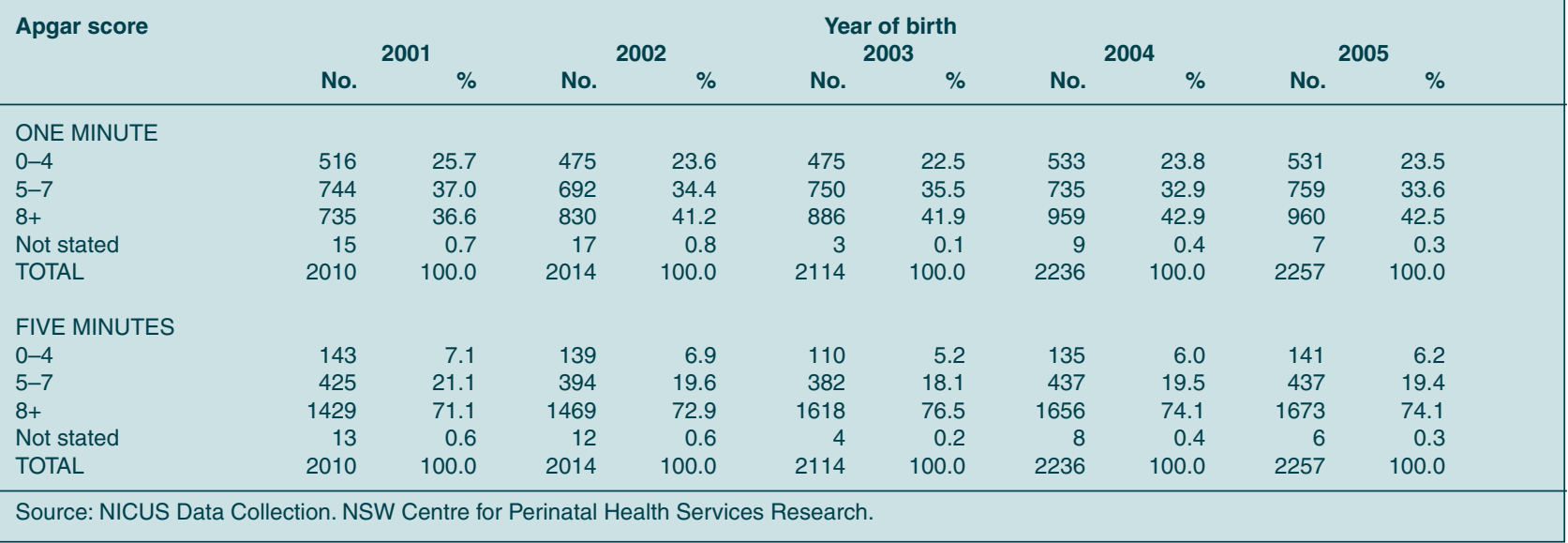

\section{TABLE 88}

NICUS REGISTRANTS BY ASSISTED VENTILATION AND GESTATIONAL AGE, NSW \& ACT 2001-2005"

\begin{tabular}{|c|c|c|c|c|c|c|c|c|c|c|c|}
\hline \multirow[t]{3}{*}{ Year } & \multirow[t]{3}{*}{ Assisted ventilation } & \multicolumn{10}{|c|}{ Gestational age (weeks) } \\
\hline & & \multicolumn{2}{|c|}{$22-27$} & \multicolumn{2}{|c|}{ 28-31 } & \multicolumn{2}{|c|}{$32-36$} & \multicolumn{2}{|c|}{$37+$} & \multicolumn{2}{|c|}{ TOTAL } \\
\hline & & No. & $\%$ & No. & $\%$ & No. & $\%$ & No. & $\%$ & No. & $\%$ \\
\hline \multirow[t]{3}{*}{2001} & No & 2 & 0.7 & 126 & 20.7 & 61 & 11.6 & 3 & 1.1 & 192 & 11.4 \\
\hline & Yes & 265 & 99.3 & 482 & 79.3 & 464 & 88.4 & 277 & 98.9 & 1488 & 88.6 \\
\hline & TOTAL & 267 & 100.0 & 608 & 100.0 & 525 & 100.0 & 280 & 100.0 & 1680 & 100.0 \\
\hline \multirow[t]{3}{*}{2002} & No & 2 & 0.7 & 90 & 16.3 & 50 & 9.0 & 4 & 1.4 & 146 & 8.8 \\
\hline & Yes & 267 & 99.3 & 463 & 83.7 & 504 & 91.0 & 284 & 98.6 & 1518 & 91.2 \\
\hline & TOTAL & 269 & 100.0 & 553 & 100.0 & 554 & 100.0 & 288 & 100.0 & 1664 & 100.0 \\
\hline \multirow[t]{3}{*}{2003} & No & 1 & 0.4 & 104 & 17.9 & 98 & 16.0 & 75 & 20.1 & 278 & 15.4 \\
\hline & Yes & 234 & 99.6 & 476 & 82.1 & 515 & 84.0 & 298 & 79.9 & 1523 & 84.6 \\
\hline & TOTAL & 235 & 100.0 & 580 & 100.0 & 613 & 100.0 & 373 & 100.0 & 1801 & 100.0 \\
\hline \multirow[t]{3}{*}{2004} & No & 0 & 0.0 & 78 & 12.9 & 83 & 12.8 & 62 & 17.2 & 223 & 12.1 \\
\hline & Yes & 239 & 100.0 & 525 & 87.1 & 563 & 87.2 & 298 & 82.8 & 1625 & 87.9 \\
\hline & TOTAL & 239 & 100.0 & 603 & 100.0 & 646 & 100.0 & 360 & 100.0 & 1848 & 100.0 \\
\hline \multirow[t]{3}{*}{2005} & No & 1 & 0.4 & 115 & 18.5 & 90 & 13.3 & 48 & 12.6 & 254 & 13.3 \\
\hline & Yes & 225 & 99.6 & 505 & 81.5 & 588 & 86.7 & 333 & 87.4 & 1651 & 86.7 \\
\hline & TOTAL & 226 & 100.0 & 620 & 100.0 & 678 & 100.0 & 381 & 100.0 & 1905 & 100.0 \\
\hline
\end{tabular}




\section{FIGURE 8}

NICUS REGISTRANTS BY MAIN INDICATION FOR ASSISTED VENTILATION, NSW \& ACT 2005*

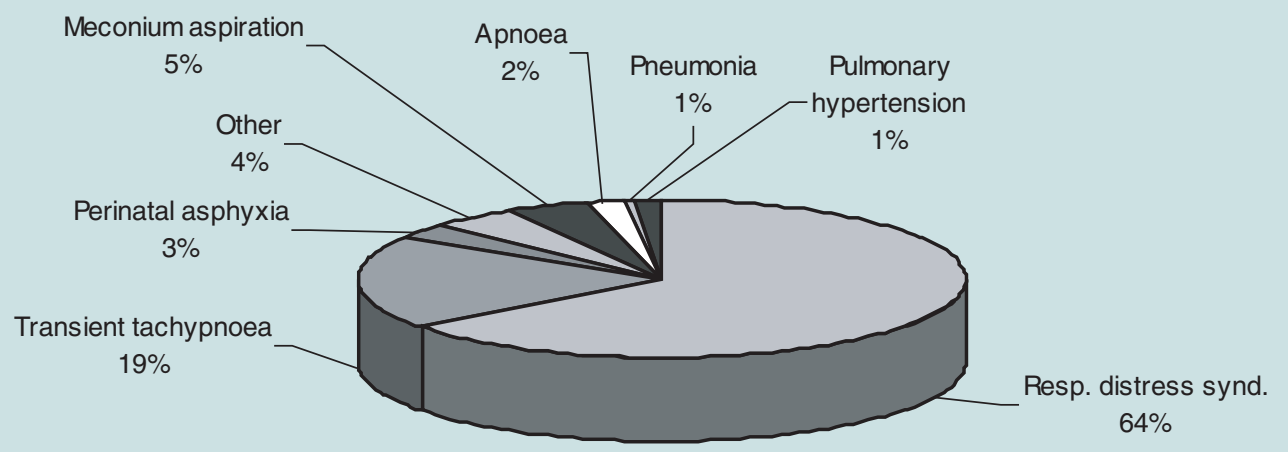

Source: NICUS Data Collection. NSW Centre for Perinatal Health Services Research.

\# Babies with major congenital anomalies or not ventilated excluded.

\section{TABLE 89}

NICUS REGISTRANTS BY MAIN INDICATION FOR ASSISTED VENTILATION AND GESTATIONAL AGE, NSW \& ACT 2005\#

\begin{tabular}{|c|c|c|c|c|c|c|c|c|c|c|}
\hline \multirow[t]{3}{*}{ Indication } & \multirow{2}{*}{\multicolumn{2}{|c|}{$22-27$}} & \multirow{2}{*}{\multicolumn{6}{|c|}{$\begin{array}{c}\text { Gestational age (weeks) } \\
\qquad 32-36\end{array}$}} & \multirow{2}{*}{\multicolumn{2}{|c|}{ TOTAL }} \\
\hline & & & & & & & & & & \\
\hline & No. & $\%$ & No. & $\%$ & No. & $\%$ & No. & $\%$ & No. & $\%$ \\
\hline Transient tachypnoea of newborn & 2 & 0.9 & 79 & 15.6 & 167 & 28.4 & 69 & 20.7 & 317 & 19.2 \\
\hline Hyaline membrane disease & 221 & 98.2 & 393 & 77.8 & 368 & 62.6 & 82 & 24.6 & 1064 & 64.4 \\
\hline Meconium aspiration & 0 & 0.0 & 1 & 0.2 & 4 & 0.7 & 70 & 21.0 & 75 & 4.5 \\
\hline Pneumonia & 0 & 0.0 & 0 & 0.0 & 3 & 0.5 & 9 & 2.7 & 12 & 0.7 \\
\hline Pulmonary hypertension & 0 & 0.0 & 2 & 0.4 & 5 & 0.9 & 15 & 4.5 & 22 & 1.3 \\
\hline Immature lung & 2 & 0.9 & 11 & 2.2 & 7 & 1.2 & 0 & 0.0 & 20 & 1.2 \\
\hline Apnoea & 0 & 0.0 & 9 & 1.8 & 12 & 2.0 & 9 & 2.7 & 30 & 1.8 \\
\hline Congenital anomaly & 0 & 0.0 & 0 & 0.0 & 2 & 0.3 & 1 & 0.3 & 3 & 0.2 \\
\hline Other & 0 & 0.0 & 5 & 1.0 & 10 & 1.7 & 25 & 7.5 & 40 & 2.4 \\
\hline Perinatal & 0 & 0.0 & 2 & 0.4 & 2 & 0.3 & 10 & 3.0 & 14 & 0.8 \\
\hline Newborn encephalopathy & 0 & 0.0 & 3 & 0.6 & 8 & 1.4 & 43 & 12.9 & 54 & 3.3 \\
\hline TOTAL & 225 & 100.0 & 505 & 100.0 & 588 & 100.0 & 333 & 100.0 & 1651 & 100.0 \\
\hline
\end{tabular}

Source: NICUS Data Collection. NSW Centre for Perinatal Health Services Research.

\# Babies with major congenital anomalies or not ventilated excluded. 


\section{TABLE 90}

NICUS REGISTRANTS BY SURFACTANT ADMINISTRATION AND GESTATIONAL AGE, NSW \& ACT 2001-2005"

\begin{tabular}{|c|c|c|c|c|c|c|c|c|c|c|c|}
\hline \multirow[t]{2}{*}{ Year } & \multirow[t]{2}{*}{$\begin{array}{l}\text { Surfactant } \\
\text { administration }\end{array}$} & \multicolumn{5}{|c|}{ Gestational age (weeks) } & $\begin{array}{l}\text { eeks) } \\
36\end{array}$ & & & & TAL \\
\hline & & No. & $\%$ & No. & $\%$ & No. & $\%$ & No. & $\%$ & No. & $\%$ \\
\hline \multirow[t]{3}{*}{2001} & No & 56 & 21.1 & 275 & 57.1 & 326 & 70.3 & 222 & 80.1 & 879 & 59.1 \\
\hline & Yes & 209 & 78.9 & 207 & 42.9 & 138 & 29.7 & 55 & 19.9 & 609 & 40.9 \\
\hline & TOTAL & 265 & 100.0 & 482 & 100.0 & 464 & 100.0 & 277 & 100.0 & 1488 & 100.0 \\
\hline \multirow[t]{3}{*}{2002} & No & 66 & 24.7 & 275 & 59.4 & 366 & 72.6 & 241 & 84.9 & 948 & 62.5 \\
\hline & Yes & 201 & 75.3 & 188 & 40.6 & 138 & 27.4 & 43 & 15.1 & 570 & 37.5 \\
\hline & TOTAL & 267 & 100.0 & 463 & 100.0 & 504 & 100.0 & 284 & 100.0 & 1518 & 100.0 \\
\hline \multirow[t]{3}{*}{2003} & No & 45 & 19.2 & 257 & 54.0 & 354 & 68.7 & 239 & 80.2 & 895 & 58.8 \\
\hline & Yes & 189 & 80.8 & 219 & 46.0 & 161 & 31.3 & 59 & 19.8 & 628 & 41.2 \\
\hline & TOTAL & 234 & 100.0 & 476 & 100.0 & 515 & 100.0 & 298 & 100.0 & 1523 & 100.0 \\
\hline \multirow[t]{3}{*}{2004} & No & 41 & 17.2 & 303 & 57.7 & 406 & 72.1 & 248 & 83.2 & 998 & 61.4 \\
\hline & Yes & 198 & 82.8 & 222 & 42.3 & 157 & 27.9 & 50 & 16.8 & 627 & 38.6 \\
\hline & TOTAL & 239 & 100.0 & 525 & 100.0 & 563 & 100.0 & 298 & 100.0 & 1625 & 100.0 \\
\hline \multirow[t]{3}{*}{2005} & No & 42 & 18.7 & 308 & 61.0 & 430 & 73.1 & 268 & 80.5 & 1048 & 63.5 \\
\hline & Yes & 183 & 81.3 & 197 & 39.0 & 158 & 26.9 & 65 & 19.5 & 603 & 36.5 \\
\hline & TOTAL & 225 & 100.0 & 505 & 100.0 & 588 & 100.0 & 333 & 100.0 & 1651 & 100.0 \\
\hline
\end{tabular}

Source: NICUS Data Collection. NSW Centre for Perinatal Health Services Research.

\# Babies with major congenital anomalies and babies not ventilated excluded.

\section{TABLE 91}

NICUS REGISTRANTS BY PROVEN SYSTEMIC INFECTION AND GESTATIONAL AGE, NSW \& ACT 2005"

Infection Gestational age (weeks)

\begin{tabular}{|c|c|c|c|c|c|c|c|c|c|c|}
\hline & \multicolumn{2}{|c|}{$22-27$} & \multicolumn{2}{|c|}{$28-31$} & \multicolumn{4}{|c|}{ 32-36 } & \multicolumn{2}{|c|}{ TOTAL } \\
\hline & No. & $\%$ & No. & $\%$ & No. & $\%$ & No. & $\%$ & No. & $\%$ \\
\hline No & 145 & 64.2 & 542 & 87.4 & 656 & 96.8 & 363 & 95.3 & 1706 & 89.6 \\
\hline Yes & 81 & 35.8 & 78 & 12.6 & 22 & 3.2 & 18 & 4.7 & 199 & 10.4 \\
\hline TOTAL & 226 & 100.0 & 620 & 100.0 & 678 & 100.0 & 381 & 100.0 & 1905 & 100.0 \\
\hline
\end{tabular}

Source: NICUS Data Collection. NSW Centre for Perinatal Health Services Research.

\# Babies with major congenital anomalies excluded.

\section{TABLE 92}

NICUS REGISTRANTS BY TREATED PATENT DUCTUS ARTERIOSUS (PDA) AND GESTATIONAL AGE, NSW \& ACT 2005\#

$\begin{array}{ll}\text { PDA-Treatment for PDA Gestational age (weeks) } & \text { Ga }\end{array}$

\begin{tabular}{|c|c|c|c|c|c|c|c|c|}
\hline \multirow[t]{3}{*}{ PDA-Treatment for PDA } & \multicolumn{8}{|c|}{ Gestational age (weeks) } \\
\hline & \multicolumn{2}{|c|}{$22-27$} & \multicolumn{2}{|c|}{ 28-31 } & \multicolumn{2}{|c|}{$32-36$} & \multicolumn{2}{|c|}{ TOTAL } \\
\hline & No. & $\%$ & No. & $\%$ & No. & $\%$ & No. & $\%$ \\
\hline No treated PDA & 103 & 45.6 & 534 & 86.1 & 670 & 98.8 & 1307 & 85.8 \\
\hline Indomethacin only & 108 & 47.8 & 76 & 12.3 & 8 & 1.2 & 192 & 12.6 \\
\hline Surgery only & 2 & 0.9 & 1 & 0.2 & 0 & 0.0 & 3 & 0.2 \\
\hline Indomethacin \& surgery & 13 & 5.8 & 9 & 1.5 & 0 & 0.0 & 22 & 1.4 \\
\hline TOTAL & 226 & 100.0 & 620 & 100.0 & 678 & 100.0 & 1524 & 100.0 \\
\hline
\end{tabular}




\section{Continued from page 66}

Overall, the incidence of necrotising enterocolitis (NEC) was 2.9 per cent in 2005 (range 9.8 per cent in 1992 to 2.2 per cent in 2000). The diagnosis of NEC was made radiologically or at surgery in 53.6 per cent of infants and clinically in the remainder. NEC was more common at the lower gestational age groups and 87.5 per cent of the infants with NEC were born at less than 32 weeks gestation (Table 93).

The overall incidence of major surgery was 3.5 per cent in 2005 (range 7.7 per cent in 1992 to 3.1 per cent in 2000). In $2005,87.5$ per cent of the infants who required major surgery were less than 32 weeks gestation (Table 94). The most common surgical procedures amongst these infants were for patent ductus arteriosus and necrotising enterocolitis.

In 2005, the incidence of intraventricular haemorrhage (IVH) among preterm infants (less than 37 weeks gestational age) was 14 per cent (range 20.5 per cent in
1993 to 12.9 per cent in 2003). In 2005, confirmed IVH was most common among infants less than 28 weeks gestation (42 per cent); 29.5 per cent of these infants had severe IVH (grade 3 or 4). Four infants less than 32 weeks gestation with severe IVH required surgical drainage for post haemorrhagic hydrocephalus (4/53, 7.5 per cent). Of the surviving infants born before 32 weeks gestation, 95 per cent had a head ultrasound examination to detect IVH (Table 95).

The proportion of infants with severe grades (Grades 3, 4 or 5 ) of retinopathy of prematurity (ROP) was 4.3 per cent in 2005 (range 7.5 per cent in 1992 to 2.8 per cent in 2004). In 2005, five infants with Grade 3 ROP were 28-31 weeks gestation and 74.2 per cent of the infants less than 28 weeks gestation with severe ROP received laser therapy. Importantly, 25.2 per cent of surviving infants of 28-31 weeks gestational age did not have an eye examination recorded (Table 96).

Continued on page 72

\section{TABLE 93}

NICUS REGISTRANTS BY NECROTISING ENTEROCOLITIS (NEC) AND GESTATIONAL AGE, NSW \& ACT $2005 *$

\begin{tabular}{|c|c|c|c|c|c|c|c|c|c|c|}
\hline \multirow{3}{*}{ NEC-Treatment for NEC } & \multicolumn{10}{|c|}{ Gestational age (weeks) } \\
\hline & \multicolumn{2}{|c|}{$22-27$} & \multicolumn{2}{|c|}{ 28-31 } & \multicolumn{2}{|c|}{$32-36$} & \multicolumn{2}{|c|}{$37+$} & \multicolumn{2}{|c|}{ TOTAL } \\
\hline & No. & $\%$ & No. & $\%$ & No. & $\%$ & No. & $\%$ & No. & $\%$ \\
\hline No NEC & 194 & 85.8 & 603 & 97.3 & 672 & 99.1 & 380 & 99.7 & 1849 & 97.1 \\
\hline Clinical diagnosis & 12 & 5.3 & 9 & 1.5 & 4 & 0.6 & 1 & 0.3 & 26 & 1.4 \\
\hline $\mathrm{X}$-ray diagnosis & 6 & 2.7 & 3 & 0.5 & 1 & 0.1 & 0 & 0.0 & 10 & 0.5 \\
\hline Surgery for NEC & 14 & 6.2 & 5 & 0.8 & 1 & 0.1 & 0 & 0.0 & 20 & 1.1 \\
\hline TOTAL & 226 & 100.0 & 620 & 100.0 & 678 & 100.0 & 381 & 100.0 & 1905 & 100.0 \\
\hline
\end{tabular}

Source: NICUS Data Collection. NSW Centre for Perinatal Health Services Research.

\# Babies with major congenital anomalies excluded.

\section{TABLE 94}

NICUS REGISTRANTS BY MAJOR SURGERY AND GESTATIONAL AGE, NSW \& ACT 2005"

\begin{tabular}{|c|c|c|c|c|c|c|c|c|c|c|}
\hline \multirow[t]{3}{*}{ Major surgery } & \multicolumn{10}{|c|}{ Gestational age (weeks) } \\
\hline & \multicolumn{2}{|c|}{$22-27$} & \multicolumn{2}{|c|}{ 28-31 } & \multicolumn{2}{|c|}{$32-36$} & \multicolumn{2}{|c|}{$37+$} & \multicolumn{2}{|c|}{ TOTAL } \\
\hline & No. & $\%$ & No. & $\%$ & No. & $\%$ & No. & $\%$ & No. & $\%$ \\
\hline No & 197 & 87.2 & 604 & 97.4 & 670 & 98.8 & 368 & 96.6 & 1839 & 96.5 \\
\hline Yes & 29 & 12.8 & 16 & 2.6 & 8 & 1.2 & 13 & 3.4 & 66 & 3.5 \\
\hline TOTAL & 226 & 100.0 & 620 & 100.0 & 678 & 100.0 & 381 & 100.0 & 1905 & 100.0 \\
\hline
\end{tabular}

Source: NICUS Data Collection. NSW Centre for Perinatal Health Services Research.

\# Babies with major congenital anomalies excluded. 
TABLE 95

NICUS REGISTRANTS BY INTRAVENTRICULAR HAEMORRHAGE (IVH) AND GESTATIONAL AGE, NSW \& ACT 2005*

Head ultrasound Gestational age (weeks)

\begin{tabular}{|c|c|c|c|c|c|c|c|c|}
\hline \multirow[t]{3}{*}{ Head ultrasound } & \multicolumn{8}{|c|}{ Gestational age (weeks) } \\
\hline & \multicolumn{2}{|c|}{$22-27$} & \multicolumn{2}{|c|}{$28-31$} & \multicolumn{2}{|c|}{$32-36$} & \multicolumn{2}{|c|}{ TOTAL } \\
\hline & No. & $\%$ & No. & $\%$ & No. & $\%$ & No. & $\%$ \\
\hline No IVH & 121 & 50.8 & 485 & 80.7 & 250 & 38.7 & 856 & 57.6 \\
\hline Grade 1 & 43 & 18.1 & 59 & 9.8 & 19 & 2.9 & 121 & 8.1 \\
\hline Grade 2 & 21 & 8.8 & 6 & 1.0 & 2 & 0.3 & 29 & 2.0 \\
\hline Grade 3 & 15 & 6.3 & 10 & 1.7 & 2 & 0.3 & 27 & 1.8 \\
\hline Grade 4 & 25 & 10.5 & 3 & 0.5 & 1 & 0.2 & 29 & 2.0 \\
\hline Hydrocephalus requiring drainage & 3 & 1.3 & 1 & 0.2 & 2 & 0.3 & 6 & 0.4 \\
\hline Not examined \& lived & 0 & 0.0 & 35 & 5.8 & 370 & 57.3 & 405 & 27.3 \\
\hline Not examined \& died & 13 & 5.5 & 3 & 0.5 & 2 & 0.3 & 18 & 1.2 \\
\hline TOTAL & 238 & 100.0 & 601 & 100.0 & 646 & 100.0 & 1485 & 100.0 \\
\hline
\end{tabular}

Source: NICUS Data Collection. NSW Centre for Perinatal Health Services Research.

\# Babies with major congenital anomalies excluded.

\section{TABLE 96}

NICUS REGISTRANTS BY RETINOPATHY OF PREMATURITY (ROP) AND GESTATIONAL AGE, NSW \& ACT 2005\#

Retinopathy of prematurity (ROP)

Gestational age (weeks)

\begin{tabular}{|c|c|c|c|c|c|c|}
\hline \multirow[t]{3}{*}{ patny or prematurity (RU) } & \multicolumn{6}{|c|}{ nal age } \\
\hline & \multicolumn{2}{|c|}{ 22-27 } & \multicolumn{2}{|c|}{ 28-31 } & \multicolumn{2}{|c|}{ TOTAL } \\
\hline & No. & $\%$ & No. & $\%$ & No. & $\%$ \\
\hline No ROP & 64 & 28.3 & 415 & 66.9 & 479 & 56.6 \\
\hline Grade 1 & 37 & 16.4 & 13 & 2.1 & 50 & 5.9 \\
\hline Grade 2 & 34 & 15.0 & 13 & 2.1 & 47 & 5.6 \\
\hline Grade 3 & 28 & 12.4 & 5 & 0.8 & 33 & 3.9 \\
\hline Grade 4 & 1 & 0.4 & 0 & 0.0 & 1 & 0.1 \\
\hline Grade 5 & 2 & 0.9 & 0 & 0.0 & 2 & 0.2 \\
\hline Treatment with laser therapy & 23 & 10.2 & 3 & 0.5 & 26 & 3.1 \\
\hline Not examined \& lived & 2 & 0.9 & 156 & 25.2 & 158 & 18.7 \\
\hline Not examined \& died & 58 & 25.7 & 18 & 3.1 & 76 & 9.0 \\
\hline TOTAL & 226 & 100.0 & 620 & 100.0 & 846 & 100.0 \\
\hline
\end{tabular}

Source: NICUS Data Collection. NSW Centre for Perinatal Health Services Research.

\# Babies with major congenital anomalies excluded. 


\section{(Continued from page 70)}

\section{Service utilisation}

Indicators of service utilisation collected as part of NICUS include length of stay in tertiary and non-tertiary centres, days on assisted ventilation, and days in oxygen (Figures 9, 10 and 11 and Table 97). On an individual basis, infants born at less than 28 weeks gestation consumed most resources. However, as a group those born at 28-31 weeks gestation consumed more bed days than any other group. In 2005, the total cohort used 62,761 bed days in a tertiary centre in NSW and the ACT (range 46,090 in 1993 to 62,852 in 2004); as well as 19,497 in a non-tertiary centre (level 2 neonatal unit) in NSW and the ACT $(14,288$ in
1992 to 21,352 in 2004). Even when these infants leave the neonatal intensive care unit, they still require substantial resources.

In 2005, NICUS registrants used 17,750 days of assisted ventilation (range 15,282 in 1993 to 18,557 in 2004) and 24,436 days of oxygen therapy (range 22,526 in 1992 to 30,802 in 2001). In 2005, 44 (2.3 per cent) infants were discharged home on oxygen therapy (range 2.1 per cent in 1992 to 5.1 per cent in 1998). The proportion of infants less than 28 weeks gestation discharged home on oxygen therapy was 10.6 per cent (range 7.5 per cent in1992 to 21.3 per cent in 2002) (Table 98).

Continued on page 76

\section{FIGURE 9}

NICUS REGISTRANTS BY TOTAL NUMBER OF DAYS IN HOSPITAL AND GESTATIONAL AGE, NSW \& ACT 2005

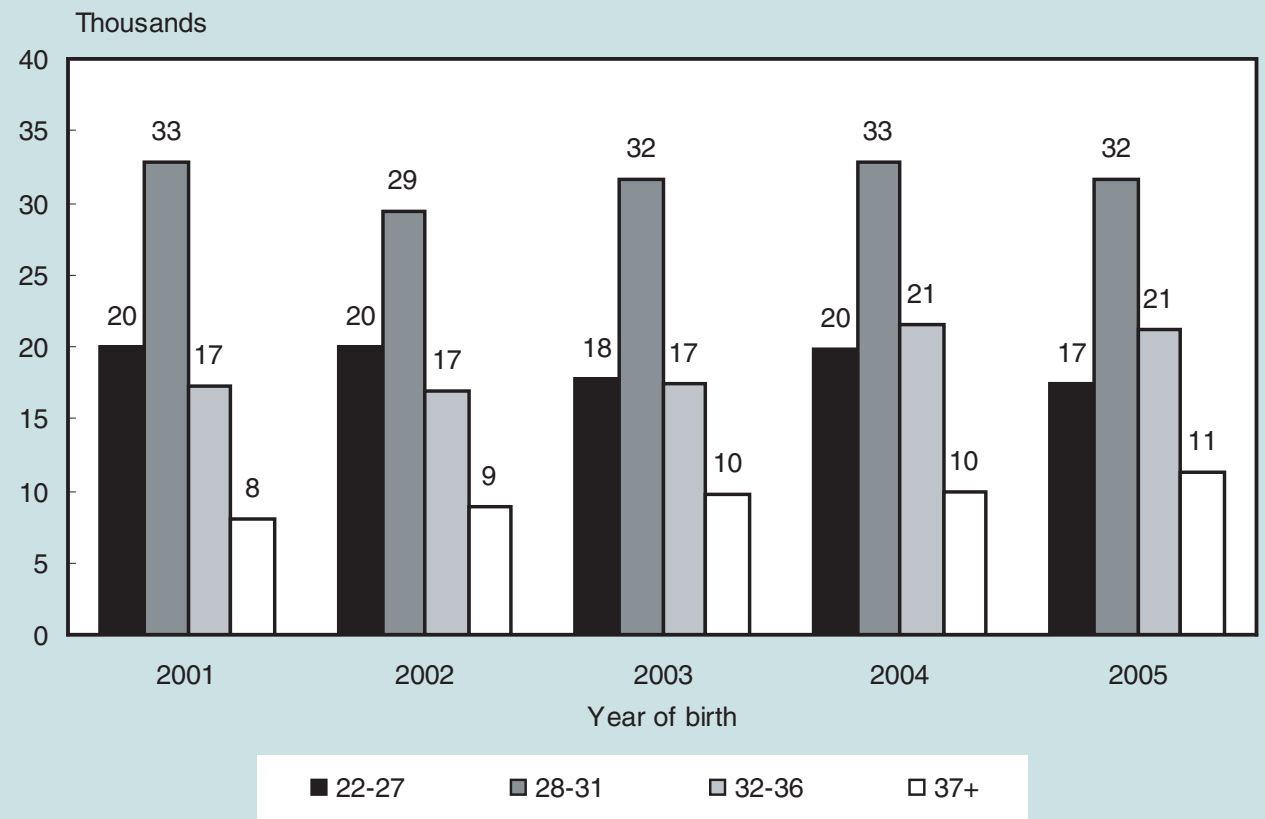

Source: NICUS Data Collection. NSW Centre for Perinatal Health Services Research. 


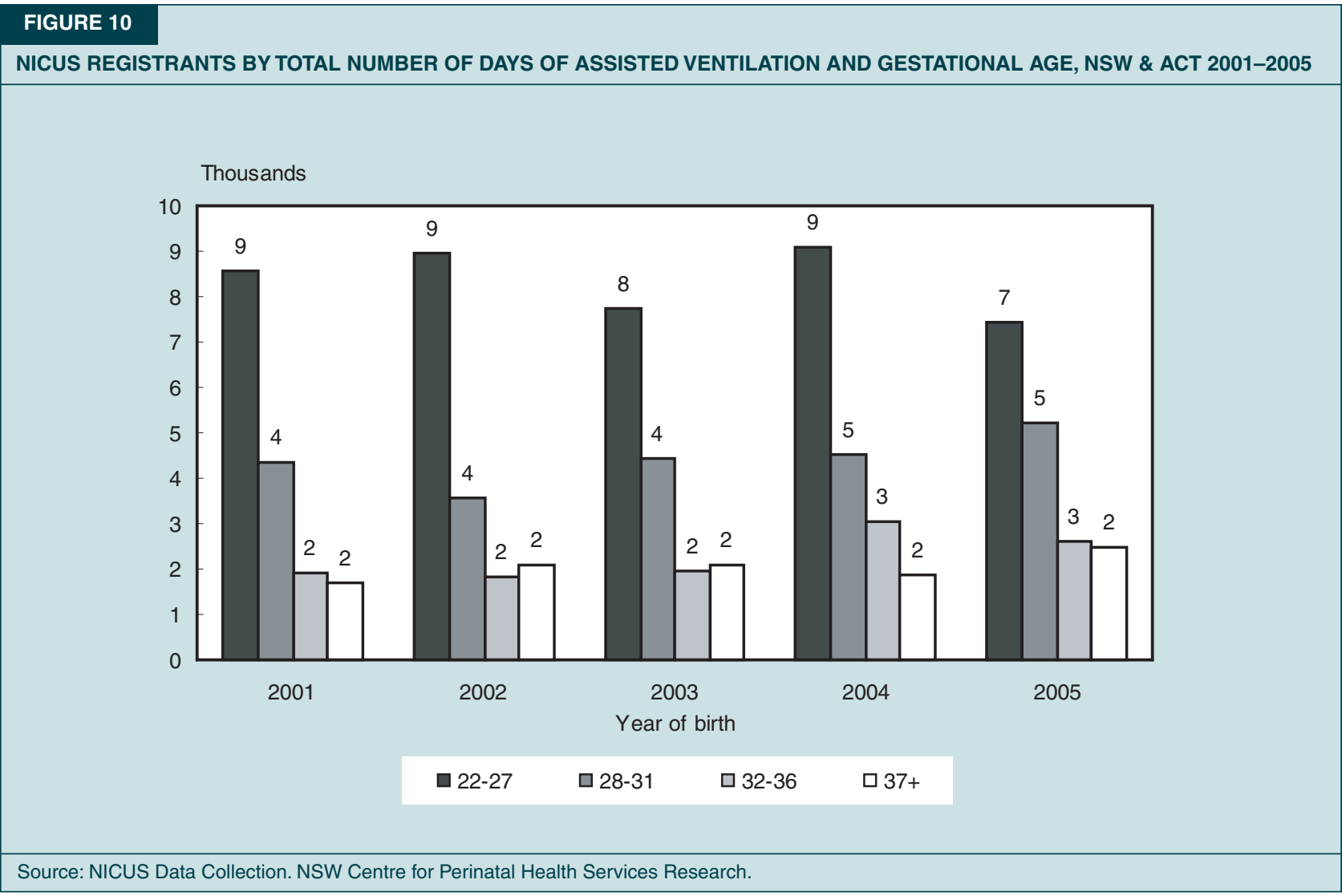

\section{FIGURE 11}

NICUS REGISTRANTS BY TOTAL NUMBER OF DAYS OF OXYGEN THERAPY AND GESTATIONAL AGE, NSW \& ACT 2001-2005

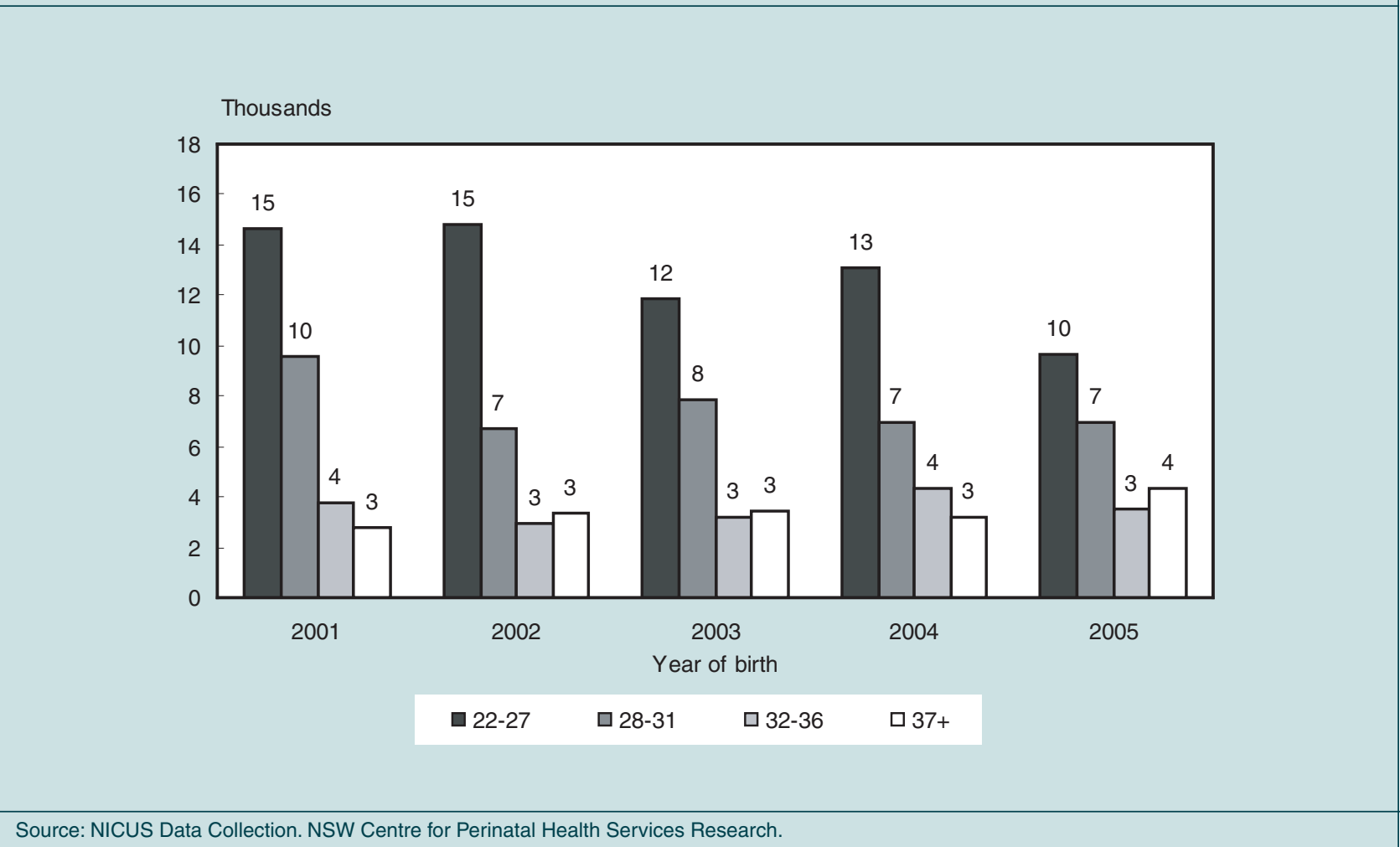




\section{TABLE 97}

NICUS REGISTRANTS BY SERVICE UTILISATION INDICATORS AND GESTATIONAL AGE, NSW \& ACT 2005

\begin{tabular}{|c|c|c|c|c|c|}
\hline \multirow[t]{2}{*}{ Indicators } & \multicolumn{5}{|c|}{ Gestational age (weeks) } \\
\hline & $22-27$ & 28-31 & $32-36$ & $37+$ & TOTAL \\
\hline \multicolumn{6}{|c|}{ Non-tertiary hospital stay (days) } \\
\hline Minimum & 0 & 0 & 0 & 0 & 0 \\
\hline Maximum & 190 & 366 & 69 & 51 & 366 \\
\hline Sum & 2218 & 9566 & 6669 & 1044 & 19497 \\
\hline Median & 0 & 13 & 4 & 0 & 0 \\
\hline 25th percentile & 0 & 0 & 0 & 0 & 0 \\
\hline 75th percentile & 14 & 27 & 15 & 2 & 15 \\
\hline \multicolumn{6}{|c|}{ Tertiary hospital stay (days) } \\
\hline Minimum & 1 & 0 & 0 & 0 & 0 \\
\hline Maximum & 251 & 660 & 345 & 700 & 700 \\
\hline Sum & 15252 & 22796 & 14516 & 10197 & 62761 \\
\hline Median & 66 & 31 & 12 & 9 & 16 \\
\hline 25th percentile & 27 & 15 & 7 & 5 & 7 \\
\hline 75th percentile & 91 & 47 & 22 & 17 & 36 \\
\hline \multicolumn{6}{|c|}{ Total hospital stay (days) } \\
\hline Minimum & 1 & 0 & 0 & 0 & 0 \\
\hline Maximum & 363 & 562 & 345 & 700 & 700 \\
\hline Sum & 17406 & 31602 & 21137 & 11241 & 81386 \\
\hline Median & 77 & 46 & 23 & 10 & 27 \\
\hline 25th percentile & 32 & 37 & 15 & 6 & 12 \\
\hline 75th percentile & 98 & 59 & 32 & 20 & 47 \\
\hline \multicolumn{6}{|c|}{ Mechanical ventilation (days) } \\
\hline Minimum & 0 & 0 & 0 & 0 & 0 \\
\hline Maximum & 89 & 115 & 89 & 236 & 236 \\
\hline Sum & 2309 & 1239 & 1161 & 1988 & 6696 \\
\hline Median & 3 & 0 & 0 & 1 & 0 \\
\hline 25th percentile & 1 & 0 & 0 & 0 & 0 \\
\hline 75th percentile & 12 & 1 & 1 & 3 & 2 \\
\hline \multicolumn{6}{|c|}{ Continuous Positive Airways Pressure (days) } \\
\hline Minimum & 0 & 0 & 0 & 0 & 0 \\
\hline Maximum & 77 & 224 & 139 & 32 & 224 \\
\hline Sum & 5122 & 3991 & 1458 & 482 & 11054 \\
\hline Median & 20 & 2 & 1 & 0 & 1 \\
\hline 25th percentile & 3 & 0 & 0 & 0 & 0 \\
\hline 75th percentile & 36 & 7 & 2 & 1 & 4 \\
\hline \multicolumn{6}{|c|}{ Assisted ventilation (days) } \\
\hline Minimum & 0 & 0 & 0 & 0 & 0 \\
\hline Maximum & 115 & 297 & 154 & 256 & 297 \\
\hline Sum & 7430 & 5230 & 2619 & 2471 & 17750 \\
\hline Median & 27 & 3 & 1 & 2 & 2 \\
\hline 25th percentile & 8 & 1 & 0 & 0 & 1 \\
\hline 75th percentile & 46 & 8 & 3 & 4 & 6 \\
\hline \multicolumn{6}{|l|}{ Oxygen (days) } \\
\hline Minimum & 0 & 0 & 0 & 0 & 0 \\
\hline Maximum & 324 & 365 & 177 & 534 & 534 \\
\hline Sum & 9681 & 6918 & 3482 & 4355 & 24436 \\
\hline Median & 19 & 2 & 1 & 2 & 2 \\
\hline 25th percentile & 4 & 1 & 1 & 1 & 1 \\
\hline 75th percentile & 66 & 7 & 4 & 6 & 7 \\
\hline
\end{tabular}




\section{TABLE 98}

NICUS REGISTRANTS BY HOME OXYGEN ADMINISTRATION AND GESTATIONAL AGE, NSW \& ACT 2001-2005"

\begin{tabular}{|c|c|c|c|c|c|c|c|c|c|c|c|}
\hline \multirow[t]{3}{*}{ Year } & \multirow[t]{3}{*}{ Home oxygen } & \multicolumn{10}{|c|}{ Gestational age (weeks) } \\
\hline & & \multicolumn{2}{|c|}{$22-27$} & \multicolumn{2}{|c|}{$28-31$} & \multicolumn{2}{|c|}{$32-36$} & \multicolumn{2}{|c|}{$37+$} & \multicolumn{2}{|c|}{ TOTAL } \\
\hline & & No. & $\%$ & No. & $\%$ & No. & $\%$ & No. & $\%$ & No. & $\%$ \\
\hline \multirow[t]{3}{*}{2001} & No & 217 & 81.3 & 579 & 95.2 & 524 & 99.8 & 277 & 98.9 & 1597 & 95.1 \\
\hline & Yes & 50 & 18.7 & 29 & 4.8 & 1 & 0.2 & 3 & 1.1 & 83 & 4.9 \\
\hline & TOTAL & 267 & 100.0 & 608 & 100.0 & 525 & 100.0 & 280 & 100.0 & 1680 & 100.0 \\
\hline \multirow[t]{3}{*}{2002} & No & 211 & 78.4 & 540 & 97.6 & 551 & 99.5 & 283 & 98.3 & 1585 & 95.3 \\
\hline & Yes & 58 & 21.6 & 13 & 2.4 & 3 & 0.5 & 5 & 1.7 & 79 & 4.7 \\
\hline & TOTAL & 269 & 100.0 & 553 & 100.0 & 554 & 100.0 & 288 & 100.0 & 1664 & 100.0 \\
\hline \multirow[t]{3}{*}{2003} & No & 200 & 85.1 & 556 & 95.9 & 611 & 99.7 & 371 & 99.5 & 1738 & 96.5 \\
\hline & Yes & 35 & 14.9 & 24 & 4.1 & 2 & 0.3 & 2 & 0.5 & 63 & 3.5 \\
\hline & TOTAL & 235 & 100.0 & 580 & 100.0 & 613 & 100.0 & 373 & 100.0 & 1801 & 100.0 \\
\hline \multirow[t]{3}{*}{2004} & No & 194 & 81.2 & 591 & 98.0 & 644 & 99.7 & 358 & 99.4 & 1787 & 96.7 \\
\hline & Yes & 45 & 18.8 & 12 & 2.0 & 2 & 0.3 & 2 & 0.6 & 61 & 3.3 \\
\hline & TOTAL & 239 & 100.0 & 603 & 100.0 & 646 & 100.0 & 360 & 100.0 & 1848 & 100.0 \\
\hline \multirow[t]{3}{*}{2005} & No & 202 & 89.4 & 603 & 97.3 & 677 & 99.9 & 379 & 99.5 & 1861 & 97.7 \\
\hline & Yes & 24 & 10.6 & 17 & 2.7 & 1 & 0.1 & 2 & 0.5 & 44 & 2.3 \\
\hline & TOTAL & 226 & 100.0 & 620 & 100.0 & 678 & 100.0 & 381 & 100.0 & 1905 & 100.0 \\
\hline
\end{tabular}




\section{(Continued from page 72)}

\section{Survival}

Infants with a major congenital anomaly have been excluded from the analysis of survival, with the exception of data reported in Table 102.

The six-month survival rate for all infants without a major congenital anomaly in the 2005 cohort was 94 per cent (range 87.8 per cent in 1992 to 94.4 per cent in 2004). Survival of infants born at less than 25 weeks gestation was 38.3 per cent (range 33.3 per cent in 2003 to 54.8 per cent in 1993). There was a trend for survival to improve with gestational age (Figure 12 and Table 99). There was no difference in the survival rate between term $(94.8$ per cent) and preterm infants (93.8 per cent). Among infants who died, 68.4 per cent of deaths occurred during the first week of life (range 62.5 per cent in 1998 to 76.2 per cent in 2002) with a further 21.1 per cent occurring during the first month of life (Table 99).

The six-month survival rate improved with increasing birth weight, ranging from 31.3 per cent for infants in the 500-599 gram group to 93.5 per cent for the 900-999 gram group. Six-month survival continued to improve with increasing birth weight to a maximum of 98.7 per cent for infants of 1,500-1,749 grams birth weight and then decreased slightly (Table 100).

The majority of infants registered in NICUS were born at a tertiary centre. Although the gestational age is the most important risk factor for mortality, disease severity is also important. At each gestational age group those with severe disease are more likely to be transferred to a neonatal intensive care unit.

In 2005, the six-month survival rate for 22-27 week infants born in a tertiary centre (74.4 per cent) was significantly higher than for those born in a non-tertiary centre (60.9 per cent). Place of birth did not significantly affect survival for infants in any of the other gestational age groups (Table 101).

The six-month survival rate was similar for males $(94.4$ per cent) and females ( 93.5 per cent) overall, and for all gestational age groups: less than 28 weeks ( 70.3 per cent versus 75.9 per cent); $28-31$ weeks (96.5 per cent versus 95.9 per cent); 32-36 weeks (98.5 per cent versus 98.6 per cent); and 37-41 weeks gestation groups (96.2 percent versus 92 per cent).

The six-month survival rate was 94 per cent $(n=1,384)$ for singleton infants and 93.8 per cent $(n=433)$ for multiple gestation infants. In 2005 plurality did not significantly influence survival in any of the gestational age groups.

As expected the overall survival rate was generally lower (92.3 per cent) in the presence of a major congenital anomaly (Table 102).

Post-mortem examinations were performed on 29/114 infants (25.4 per cent) who died in the 2005 cohort (Figure 13 and Table 103). Post-mortem examinations were

\section{FIGURE 12}

\section{NICUS REGISTRANTS BY 6-MONTHS SURVIVAL AND GESTATIONAL AGE, NSW \& ACT 2001-2005"}

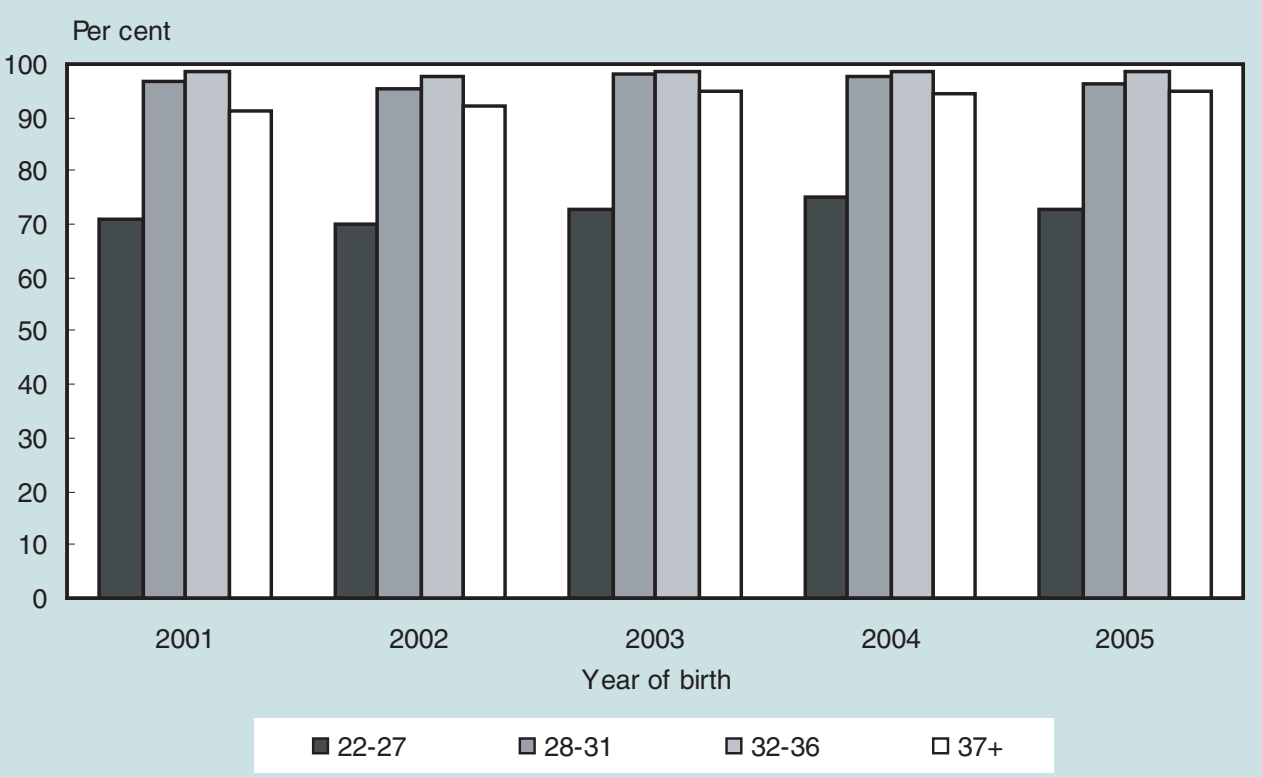

Source: NICUS Data Collection. NSW Centre for Perinatal Health Services Research.

\# Babies with major congenital anomalies excluded. 
most commonly not requested for infants 22-27 weeks gestation (57.4 per cent). The highest rate of refusal was in the 28-31 week group (30.4 per cent) and the highest rate of post-mortems done was in the 32-36 week (60 per cent).

\begin{tabular}{|c|c|c|c|c|c|c|c|c|c|c|}
\hline \multirow[t]{3}{*}{ Gestational age (weeks) } & \multirow{2}{*}{\multicolumn{2}{|c|}{$\begin{array}{c}\text { Alive at } 6 \\
\text { months }\end{array}$}} & \multicolumn{8}{|c|}{ Age at death (days) } \\
\hline & & & & $0-7$ & & & & & & TTAL \\
\hline & No. & $\%$ & No. & $\%$ & No. & $\%$ & No. & $\%$ & No. & $\%$ \\
\hline 22 & 0 & 0.0 & 1 & 100.0 & 0 & 0.0 & 0 & 0.0 & 1 & 0.1 \\
\hline 23 & 2 & 28.6 & 4 & 57.1 & 1 & 14.3 & 0 & 0.0 & 7 & 0.4 \\
\hline 24 & 16 & 41.0 & 16 & 41.0 & 4 & 10.3 & 3 & 7.7 & 39 & 2.0 \\
\hline 25 & 31 & 66.0 & 11 & 23.4 & 3 & 6.4 & 2 & 4.3 & 47 & 2.5 \\
\hline 26 & 50 & 84.7 & 6 & 10.2 & 3 & 5.1 & 0 & 0.0 & 59 & 3.1 \\
\hline 27 & 66 & 90.4 & 4 & 5.5 & 2 & 2.7 & 1 & 1.4 & 73 & 3.8 \\
\hline 28 & 107 & 93.9 & 5 & 4.4 & 1 & 0.9 & 1 & 0.9 & 114 & 6.0 \\
\hline 29 & 106 & 93.0 & 4 & 3.5 & 3 & 2.6 & 1 & 0.9 & 114 & 6.0 \\
\hline 30 & 158 & 97.5 & 1 & 0.6 & 1 & 0.6 & 2 & 1.2 & 162 & 8.5 \\
\hline 31 & 226 & 98.3 & 4 & 1.7 & 0 & 0.0 & 0 & 0.0 & 230 & 12.1 \\
\hline 32 & 198 & 97.5 & 3 & 1.5 & 0 & 0.0 & 2 & 1.0 & 203 & 10.7 \\
\hline 33 & 144 & 98.6 & 2 & 1.4 & 0 & 0.0 & 0 & 0.0 & 146 & 7.7 \\
\hline 34 & 139 & 98.6 & 2 & 1.4 & 0 & 0.0 & 0 & 0.0 & 141 & 7.4 \\
\hline 35 & 98 & 99.0 & 1 & 1.0 & 0 & 0.0 & 0 & 0.0 & 99 & 5.2 \\
\hline 36 & 89 & 100.0 & 0 & 0.0 & 0 & 0.0 & 0 & 0.0 & 89 & 4.7 \\
\hline 37 & 61 & 98.4 & 0 & 0.0 & 1 & 1.6 & 0 & 0.0 & 62 & 3.3 \\
\hline 38 & 82 & 93.2 & 5 & 5.7 & 1 & 1.1 & 0 & 0.0 & 88 & 4.6 \\
\hline 39 & 70 & 97.2 & 1 & 1.4 & 1 & 1.4 & 0 & 0.0 & 72 & 3.8 \\
\hline 40 & 85 & 93.4 & 5 & 5.5 & 1 & 1.1 & 0 & 0.0 & 91 & 4.8 \\
\hline 41 & 56 & 91.8 & 3 & 4.9 & 2 & 3.3 & 0 & 0.0 & 61 & 3.2 \\
\hline 42 & 7 & 100.0 & 0 & 0.0 & 0 & 0 & 0 & 0.0 & 7 & 0.4 \\
\hline TOTAL & 1791 & 94.0 & 78 & 4.1 & 24 & 1.3 & 12 & 0.6 & 1905 & 100.0 \\
\hline
\end{tabular}

\section{TABLE 100}

NICUS REGISTRANTS BY DURATION OF SURVIVAL AND BIRTH WEIGHT, NSW \& ACT 2005\#

\begin{tabular}{|c|c|c|c|c|c|c|c|c|c|c|}
\hline \multirow[t]{3}{*}{ Birth weight (grams) } & \multirow{2}{*}{\multicolumn{2}{|c|}{$\begin{array}{l}\text { Alive at } 5 \\
\text { months }\end{array}$}} & \multicolumn{8}{|c|}{ Age at death (days) } \\
\hline & & & \multicolumn{2}{|c|}{$0-7$} & \multicolumn{2}{|c|}{$8-28$} & \multicolumn{2}{|c|}{$28+$} & \multicolumn{2}{|c|}{ TOTAL } \\
\hline & No. & $\%$ & No. & $\%$ & No. & $\%$ & No. & $\%$ & No. & $\%$ \\
\hline Less than 400 & 0 & 0.0 & 0 & 0.0 & 0 & 0.0 & 0 & 0.0 & 0 & 0.0 \\
\hline $400-499$ & 2 & 40.0 & 3 & 60.0 & 0 & 0.0 & 0 & 0.0 & 5 & 0.3 \\
\hline $500-599$ & 5 & 31.3 & 7 & 43.8 & 2 & 12.5 & 2 & 12.5 & 16 & 0.8 \\
\hline $600-699$ & 25 & 50.0 & 17 & 34.0 & 5 & 10.0 & 3 & 6.0 & 50 & 2.6 \\
\hline $700-799$ & 33 & 73.3 & 9 & 20.0 & 2 & 4.4 & 1 & 2.2 & 45 & 2.4 \\
\hline $800-899$ & 39 & 84.8 & 4 & 8.7 & 2 & 4.3 & 1 & 2.2 & 46 & 2.4 \\
\hline $900-999$ & 58 & 93.5 & 3 & 4.8 & 1 & 1.6 & 0 & 0.0 & 62 & 3.3 \\
\hline $1,000-1,249$ & 210 & 94.6 & 5 & 2.3 & 5 & 2.3 & 2 & 0.9 & 222 & 11.7 \\
\hline $1,250-1,499$ & 226 & 97.0 & 5 & 2.2 & 1 & 0.4 & 1 & 0.4 & 233 & 12.2 \\
\hline $1,500-1,749$ & 227 & 98.7 & 2 & 0.9 & 0 & 0.0 & 1 & 0.4 & 230 & 12.1 \\
\hline $1,750-1,999$ & 184 & 97.4 & 4 & 2.1 & 0 & 0.0 & 1 & 0.5 & 189 & 9.9 \\
\hline $2,000-2,499$ & 260 & 97.4 & 7 & 2.6 & 0 & 0.0 & 0 & 0.0 & 267 & 14.0 \\
\hline $2,500-2,999$ & 172 & 97.7 & 3 & 1.7 & 1 & 0.6 & 0 & 0.0 & 176 & 9.2 \\
\hline $3,000-3,499$ & 180 & 97.8 & 1 & 0.5 & 3 & 1.6 & 0 & 0.0 & 184 & 9.7 \\
\hline $3,500-3,999$ & 98 & 93.3 & 6 & 5.7 & 1 & 1.0 & 0 & 0.0 & 105 & 5.5 \\
\hline $4,000+$ & 72 & 96.0 & 2 & 2.7 & 1 & 1.3 & 0 & 0.0 & 75 & 3.9 \\
\hline TOTAL & 1791 & 94.0 & 78 & 4.1 & 24 & 1.3 & 12 & 0.6 & 1905 & 100.0 \\
\hline
\end{tabular}


TABLE 101

NICUS REGISTRANTS BY DURATION OF SURVIVAL, PLACE OF BIRTH AND GESTATIONAL AGE, NSW \& ACT 2005*

\begin{tabular}{|c|c|c|c|c|c|c|c|c|c|c|c|}
\hline \multirow{3}{*}{$\begin{array}{l}\text { Gestational } \\
\text { age (weeks) }\end{array}$} & \multirow{3}{*}{$\begin{array}{l}\text { Place of } \\
\text { birth }\end{array}$} & \multirow{2}{*}{\multicolumn{2}{|c|}{$\begin{array}{c}\text { Alive at } 6 \\
\text { months }\end{array}$}} & \multicolumn{8}{|c|}{ Age at death (days) } \\
\hline & & & & & & & & & & & TAL \\
\hline & & No. & $\%$ & No. $\%$ & No. & $\%$ & No. & $\%$ & No. & $\%$ & \\
\hline \multirow[t]{3}{*}{$22-27$} & Non tertiary & 14 & 60.9 & 7 & 30.4 & 1 & 4.3 & 1 & 4.3 & 23 & 10.2 \\
\hline & Tertiary & 151 & 74.4 & 35 & 17.2 & 12 & 5.9 & 5 & 2.5 & 203 & 89.8 \\
\hline & Sub-total & 165 & 73.0 & 42 & 18.6 & 13 & 5.8 & 6 & 2.7 & 226 & 100.0 \\
\hline \multirow[t]{3}{*}{$28-31$} & Non tertiary & 65 & 100.0 & 0 & 0.0 & 0 & 0.0 & 0 & 0.0 & 65 & 10.5 \\
\hline & Tertiary & 530 & 95.8 & 14 & 2.5 & 5 & 0.9 & 4 & 0.7 & 553 & 89.5 \\
\hline & Sub-total & 595 & 96.3 & 14 & 2.3 & 5 & 0.8 & 4 & 0.7 & 618 & 100.0 \\
\hline \multirow[t]{3}{*}{$32-36$} & Non tertiary & 186 & 98.4 & 3 & 1.6 & 0 & 0.0 & 0 & 0.0 & 189 & 28.0 \\
\hline & Tertiary & 479 & 98.8 & 4 & 0.8 & 0 & 0.0 & 2 & 0.4 & 485 & 72.0 \\
\hline & Sub-total & 665 & 98.7 & 7 & 1.0 & 0 & 0.0 & 2 & 0.3 & 674 & 100.0 \\
\hline \multirow[t]{3}{*}{$37-41$} & Non tertiary & 190 & 93.1 & 10 & 4.9 & 4 & 2.0 & 0 & 0.0 & 204 & 54.8 \\
\hline & Tertiary & 162 & 96.4 & 4 & 2.4 & 2 & 1.2 & 0 & 0.0 & 168 & 45.2 \\
\hline & Sub-total & 352 & 94.6 & 14 & 3.8 & 6 & 1.6 & 0 & 0.0 & 372 & 100.0 \\
\hline \multirow[t]{2}{*}{$42+$} & Tertiary & 6 & 100.0 & 0 & 0.0 & 0 & 0.0 & 0 & 0.0 & 6 & 100.0 \\
\hline & Sub-total & 6 & 100.0 & 0 & 0.0 & 0 & 0.0 & 0 & 0.0 & 6 & 100.0 \\
\hline TOTAL & & 1783 & 94.0 & 77 & 4.1 & 24 & 1.3 & 12 & 0.6 & 1896 & 100.0 \\
\hline
\end{tabular}

\section{TABLE 102}

NICUS REGISTRANTS BY DURATION OF SURVIVAL, MAJOR CONGENITAL ANOMALY AND GESTATIONAL AGE, NSW \& ACT 2005

\begin{tabular}{|c|c|c|c|c|c|c|c|c|c|c|c|}
\hline \multirow{3}{*}{$\begin{array}{l}\text { Gestational } \\
\text { age (weeks) }\end{array}$} & \multirow{3}{*}{$\begin{array}{l}\text { Major congenital } \\
\text { anomaly }\end{array}$} & \multirow{2}{*}{\multicolumn{2}{|c|}{$\begin{array}{c}\text { Alive at } 6 \\
\text { months }\end{array}$}} & \multicolumn{8}{|c|}{ Age at death (days) } \\
\hline & & & & & & & & & & & TAL \\
\hline & & No. & $\%$ & No. & $\%$ & No. & $\%$ & No. & $\%$ & No. & $\%$ \\
\hline \multirow[t]{3}{*}{$22-27$} & No & 165 & 73.0 & 42 & 18.6 & 13 & 5.8 & 6 & 2.7 & 226 & 93.0 \\
\hline & Yes & 14 & 82.4 & 3 & 17.6 & 0 & 0.0 & 0 & 0.0 & 17 & 7.0 \\
\hline & Sub-total & 179 & 73.7 & 45 & 18.5 & 13 & 5.3 & 6 & 2.5 & 243 & 100.0 \\
\hline \multirow[t]{3}{*}{$28-31$} & No & 597 & 96.3 & 14 & 2.3 & 5 & 0.8 & 4 & 0.6 & 620 & 95.1 \\
\hline & Yes & 21 & 65.6 & 6 & 18.8 & 3 & 9.4 & 2 & 6.3 & 32 & 4.9 \\
\hline & Sub-total & 618 & 94.8 & 20 & 3.1 & 8 & 1.2 & 6 & 0.9 & 652 & 100.0 \\
\hline \multirow[t]{3}{*}{$32-36$} & No & 668 & 98.5 & 8 & 1.2 & 0 & 0.0 & 2 & 0.3 & 678 & 87.1 \\
\hline & Yes & 81 & 81.0 & 9 & 9.0 & 7 & 7.0 & 3 & 3.0 & 100 & 12.9 \\
\hline & Sub-total & 749 & 96.3 & 17 & 2.2 & 7 & 0.9 & 5 & 0.6 & 778 & 100.0 \\
\hline \multirow[t]{3}{*}{$37-41$} & No & 354 & 94.7 & 14 & 3.7 & 6 & 1.6 & 0 & 0.0 & 374 & 64.9 \\
\hline & Yes & 175 & 86.6 & 16 & 7.9 & 9 & 4.5 & 2 & 1.0 & 202 & 35.1 \\
\hline & Sub-total & 529 & 91.8 & 30 & 5.2 & 15 & 2.6 & 2 & 0.3 & 576 & 100.0 \\
\hline \multirow[t]{3}{*}{$42+$} & No & 7 & 100.0 & 0 & 0.0 & 0 & 0.0 & 0 & 0.0 & 7 & 87.5 \\
\hline & Yes & 1 & 100.0 & 0 & 0.0 & 0 & 0.0 & 0 & 0.0 & 1 & 12.5 \\
\hline & Sub-total & 8 & 100.0 & 0 & 0.0 & 0 & 0.0 & 0 & 0.0 & 8 & 100.0 \\
\hline TOTAL & & 2083 & 92.3 & 112 & 5.0 & 43 & 1.9 & 19 & 0.8 & 2257 & 100.0 \\
\hline
\end{tabular}

Source: NICUS Data Collection. NSW Centre for Perinatal Health Services Research. 


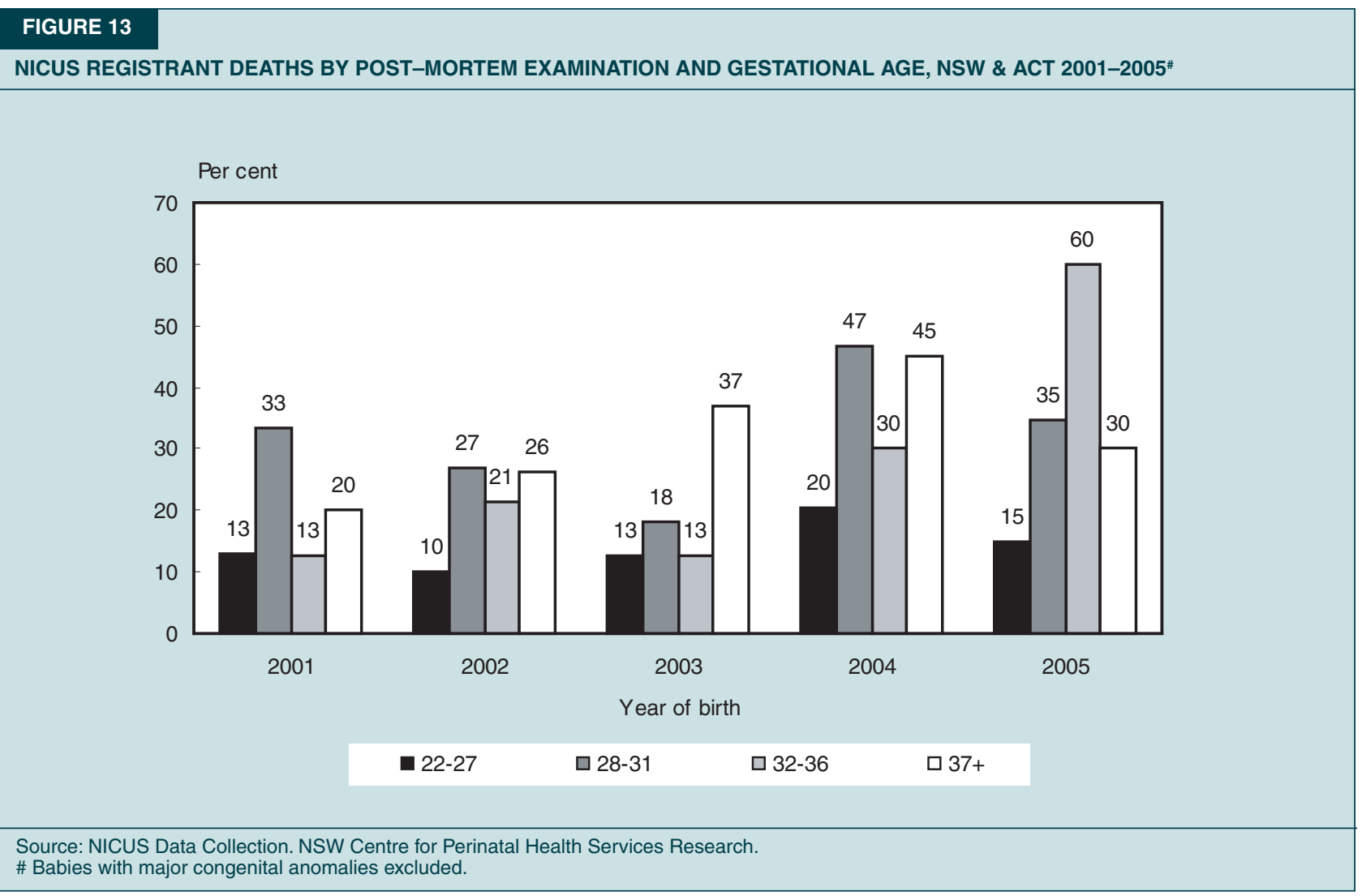

\begin{tabular}{|c|c|c|c|c|c|c|c|c|c|c|}
\hline \multirow[t]{3}{*}{ Post-mortem } & \multicolumn{10}{|c|}{ Gestational age (weeks) } \\
\hline & \multicolumn{2}{|c|}{$22-27$} & \multicolumn{2}{|c|}{ 28-31 } & \multicolumn{2}{|c|}{$32-36$} & \multicolumn{2}{|c|}{$37+$} & \multicolumn{2}{|c|}{ TOTAL } \\
\hline & No. & $\%$ & No. & $\%$ & No. & $\%$ & No. & $\%$ & No. & $\%$ \\
\hline Not requested & 35 & 57.4 & 8 & 34.8 & 2 & 20.0 & 10 & 50.0 & 55 & 48.2 \\
\hline Refused & 17 & 27.9 & 7 & 30.4 & 2 & 20.0 & 4 & 20.0 & 30 & 26.3 \\
\hline Done & 9 & 14.8 & 8 & 34.8 & 6 & 60.0 & 6 & 30.0 & 29 & 25.4 \\
\hline TOTAL & 61 & 100.0 & 23 & 100.0 & 10 & 100.0 & 20 & 100.0 & 114 & 100.0 \\
\hline
\end{tabular}

